\title{
Fast Diffusion Limit for Reaction-Diffusion Systems with Stochastic Neumann Boundary Conditions
}

\author{
Wael W. Mohammed ${ }^{1}$ and Dirk Blömker ${ }^{2}$ \\ ${ }^{1}$ Department of Mathematics, Faculty of Science, \\ Mansoura University, Egypt \\ E-mail: wael.mohammed@mans.edu.eg \\ ${ }^{2}$ Institut für Mathematik \\ Universität Augsburg, Germany \\ E-mail: dirk.bloemker@math.uni-augsburg.de
}

August 3, 2018

\begin{abstract}
We consider a class of reaction-diffusion equations with a stochastic perturbation on the boundary. We show that in the limit of fast diffusion, one can rigorously approximate solutions of the system of PDEs with stochastic Neumann boundary conditions by the solution of a suitable stochastic/deterministic differential equation for the average concentration that involves reactions only. An interesting effect occurs, if the noise on the boundary does not change the averaging concentration, but is sufficiently large. Then surprising additional effective reaction terms appear.

We focus on systems with polynomial nonlinearities only and give applications to the two dimensional nonlinear heat equation and the cubic auto-catalytic reaction between two chemicals.
\end{abstract}

Keywords: Multi-scale analysis, SPDEs, stochastic boundary conditions, reaction-diffusion equations, fast diffusion limit.

Mathematics Subject Classification: 60H10, 60H15, 35R60, 35K57.

\section{Introduction}

Stochastic partial differential equations (SPDEs) appear naturally as models for dynamical systems with respect to random influences. Sometimes in a complex physical system the noise has an impact not only on the bulk of the system but on its physical boundary, too. This happens for instance in heat transfer in a solid in contact with a fluid [11, chemical reactor theory [12, colloid and interface chemistry [19, and the air-sea interactions on the ocean surface [17].

Let $G$ be a bounded sufficiently smooth domain in $\mathbb{R}^{d}$ for $d \geq 1$, which has a smooth boundary $\partial G$. We consider the following system of stochastic reactiondiffusion equations for $n$ species with respect to random Neumann boundary 
conditions

$$
\begin{aligned}
\partial_{t} u & =\varepsilon^{-2} \mathcal{A} u+\mathcal{F}(u), \quad \text { for } t \geq 0, x \in G, \\
\frac{\partial u}{\partial \nu} & =\sigma_{\varepsilon} \partial_{t} W(t) \quad \text { for } t \geq 0, x \in \partial G, \\
u(0, x) & =u_{0}(x) \quad \text { for } \quad x \in G,
\end{aligned}
$$

with

$$
\mathcal{A} u=\left(\begin{array}{c}
\mathcal{A}_{1} u_{1} \\
\vdots \\
\mathcal{A}_{n} u_{n}
\end{array}\right), \quad \mathcal{F}(u)=\left(\begin{array}{c}
\mathcal{F}_{1}\left(u_{1}, . ., u_{n}\right) \\
\vdots \\
\mathcal{F}_{n}\left(u_{1}, . ., u_{n}\right)
\end{array}\right) \quad \text { and } W(t)=\left(\begin{array}{c}
W_{1}(t) \\
\vdots \\
W_{n}(t)
\end{array}\right) \text {, }
$$

where $\mathcal{A}$ is the diffusion term, the reaction terms $\mathcal{F}_{i}\left(u_{1}, u_{2}, \ldots, u_{n}\right)$ are polynomials of degree $m_{i}, W_{i}$ are independent $Q$-Wiener process in $\mathcal{L}^{2}(\partial G)$, and $\frac{\partial u}{\partial \nu}$ is the normal derivative of $u$ on $\partial G$. The assumption of independence is mainly for convenience of presentation, as now some terms cancel and the technicalities are less involved.

Sowers [15] investigated multidimensional stochastic reaction diffusion equation with Neumann boundary conditions and he showed that there is a unique solution. Da Prato and Zabczyk 8, 9] discussed the difference between the problems with Dirichlet and Neumann boundary noises, while [1, 3. study random Dirichlet boundary conditions. Other results are [13, 2].

An very interesting result is by Schnaubelt and Veraar [18, where regularity of solutions is studied. Furthermore, mild and weak solutions are shown to coincide.

Recently, Cerrai and Freidlin 4 considered a class of stochastic reactiondiffusion equations with Neumann boundary noise. Also, they showed that when the diffusion rate is much larger than the rate of reaction, it is possible to replace the SPDE by a suitable one-dimensional stochastic differential equation. But their result only allowed for weak convergence of the approximation without any order of the error.

Our aim is to establish rigorously error bounds results for the fast-diffusion limit for the general class of PDEs with stochastic Neumann boundary conditions given by (1). The error estimates are performed in an $\mathcal{L}^{p}$-space setting, as we cannot expect solutions to (1) to be smooth. Especially, at the forced boundary the solution $u$ is expected to be even unbounded, although it is smoother inside the domain. See [18 or for Dirichlet boundary 1 .

We consider two cases. The second on is the relatively simple limit, where the fast diffusion just disappears in the limit, while in the first case large noise changes the limiting reaction equation. The reason for large noise might be that both diffusion and noise are enhanced by stirring.

First case: If the noise does not change the average $\left(W_{c}=0\right)$ but is sufficiently large $\left(\sigma_{\varepsilon}=\varepsilon^{-1}\right)$, then the solutions of Equation (11) are well approximated by

$$
u(t, x) \simeq b(t)+\mathcal{Z}^{s}(t, x)+\text { error }
$$

where $b(t) \in \mathbb{R}^{n}$ represents the average concentration of the components of $u$ given in general formulation as a solution of

$$
\partial_{t} b(t)=\mathcal{F}(b(t))+\mathcal{G}(b(t)),
$$


for some polynomial $\mathcal{G}$ of degree less than or equal $m-2$ depending on the structure of the noise. The stochastic perturbation $\mathcal{Z}^{s}(t, x)$ is defined later in (27). It is an $\varepsilon$-dependent fast Ornstein-Uhlenbeck process (OU-process) corresponding to white noise in the limit $\varepsilon \rightarrow 0$. The index $c$ denotes the average (i.e., $v_{c}=|G|^{-1} \int_{G} v d x$ which is the projection onto the constants).

The ODE $\partial_{t} b(t)=\mathcal{F}(b)$ is the expected result, but due to noise an additional term of noise induced effective reactions appears. We illustrate our results using a relatively simple auto-catalytic reaction. For the result presented we always need a square which averages to a constant in the limit $\varepsilon \rightarrow 0$. This is mainly, because we assumed independent noise terms for each species. In contrast, if the noise terms are dependent, then any reaction term could lead to an additional effective reaction term in the limit.

Second case: If $W_{c} \neq 0$ and $\sigma_{\varepsilon}=1$, then the solution of Equation (11) are well approximated by

$$
u(t, x)=b(t)+\text { error, }
$$

and $b$ is the solution of stochastic ordinary differential equation

$$
\partial_{t} b(t)=\mathcal{F}(b(t))+\partial_{t} \tilde{\beta}(t)
$$

for some Wiener process $\tilde{\beta}$ in $\mathbb{R}^{n}$, which is essentially the projection of $W$ onto the dominant constant modes, i.e. the direct impact of the noise on the average. This is the somewhat expected result, where the reaction-diffusion equation under fast diffusion is well approximated by the reaction ODE.

As an application of our results, we give some examples from physics (nonlinear heat equation) and from chemistry (cubic auto-catalytic reaction between two chemicals according to the rule $A+B \rightarrow 2 B$ ). To illustrate our results let us focus for a moment on the relatively simple two dimensional nonlinear heat equation (also called Ginzburg-Landau or Allen-Cahn), which is partly covered by the setting of [4, too.

$$
\begin{aligned}
& \partial_{t} u=\varepsilon^{-2} \Delta u+u-u^{3} \quad \text { for } t \geq 0, x \in[0,1]^{2}, \\
& \frac{\partial u}{\partial \nu}=\sigma_{\varepsilon} \partial_{t} W(t) \quad \text { for } t \geq 0, x \in \partial[0,1]^{2} .
\end{aligned}
$$

For the first case we suppose $W_{c}=0$ and $\sigma_{\varepsilon}=\varepsilon^{-1}$, and our main Theorem 17 states that the solution of (6) is well approximated by (2) and $b$ is the solution of

$$
d b=\left[\left(1-C_{\alpha, \lambda}\right) b-b^{3}\right] d t,
$$

where $C_{\alpha, \lambda}$ is a constant depending on the noise intensity parameters $\alpha_{i, k}$ and the eigenvalues of the operator $\Delta$.

For the second case $W_{c} \neq 0$ and $\sigma_{\varepsilon}=1$ our main Theorem 20] states that the solution of (6) is of the form (4) and $b$ is the solution of

$$
d b=\left[b-b^{3}\right] d t+d B,
$$

where $B$ is a $\mathbb{R}$-valued standard Brownian motion.

The main novelties of this paper are on one hand the explicit error estimate in terms of high moments of the error, as usually only weak convergence is treated (see e.g. 4]), and on the other hand the observation that large massconservative noise has the potential to change effective reaction equations in the limit of large diffusion. 
The paper is organized as follows. Our assumptions and some definitions are given in the next section. In Section 3 we derive the fast-diffusion limit with error terms and present the main theorem. Section 4 gives bounds for high non-dominant modes, while Section 5 provides averaging results over the fast OU-process. In Section 6, we give the proof of the approximation Theorem I and some examples from physics and chemistry as applications of our results. Finally, we prove the approximation Theorem II and apply this result to nonlinear heat equation and cubic auto-catalytic reaction between two chemicals.

\section{Definition and Assumptions}

This section states the precise setting for (1) and summarizes all assumptions necessary for our results. For the analysis we work in the separable Hilbert space $\mathcal{L}^{2}(G)$ of square integrable functions, where $G \subset \mathbb{R}^{d}$ is a bounded domain with sufficiently smooth boundary $\partial G$ (e.g. Lipschitz), equipped with scalar product $\langle\cdot, \cdot\rangle$ and norm $\|\cdot\|$.

Definition 1 Define for $i=1,2, \ldots, n$ and diffusion constants $d_{i}>0$

$$
\mathcal{A}_{i}=d_{i} \Delta
$$

with

$$
D\left(\mathcal{A}_{i}\right)=\left\{u \in \mathcal{H}^{2}:\left.\partial_{\nu} u\right|_{\partial G}=0\right\},
$$

where $\partial_{\nu} u$ is the normal derivative of $u$ on $\partial G$.

Let $\left\{g_{k}\right\}_{k=1}^{\infty}$ be an orthonormal basis of eigenfunctions of $\mathcal{A}_{i}$ in $\mathcal{L}^{2}(G)$. It is obviously the same basis for all $i$ with corresponding eigenvalues $\left\{d_{i} \lambda_{k}\right\}_{k=0}^{\infty}$ depending on $i$ (cf. Courant and Hilbert [5]). Also, let $\left\{e_{k}\right\}_{k=1}^{n}$ be the standard orthonormal basis of $\mathbb{R}^{n}$. Hence, $\left\{g_{k} e_{i}\right\}$ for $k \in \mathbb{N}_{0}$ and $i=\{1, \ldots, n\}$, is an orthonormal basis of $\mathcal{A}$ in $\left[\mathcal{L}^{2}(G)\right]^{n}$ such that $\mathcal{A}\left(g_{k} e_{i}\right)=-d_{i} \lambda_{k} g_{k} e_{i}$.

Assumption 2 We assume that for all $k \in \mathbb{N}$

$$
\left\|g_{k}\right\|_{\infty} \leq C \lambda_{k}^{\gamma_{1}} \quad \text { for some } \gamma_{1} \geq 0
$$

This is true in $\mathbb{R}^{2}$ for instance on squares, hexagons, and triangles with $\gamma_{1}=0$, while the worst case is $\gamma_{1}=(d-1) / 2$ realized for balls and spheres. See [6]. This condition might be relaxed, but we focused in examples mainly on cases with $\gamma_{1}=0$.

Define

$$
\mathcal{N}:=\operatorname{ker} \mathcal{A}=\operatorname{span}\left\{e_{1} g_{0}, \ldots, e_{n} g_{0}\right\}
$$

where $g_{0}=|G|^{-\frac{1}{2}}$ is a constant and $\lambda_{0}=0$. Define $S=\mathcal{N}^{\perp}$ to be the orthogonal complement of $\mathcal{N}$ in $\left[\mathcal{L}^{2}(G)\right]^{n}$. Denote by $P_{c} u=\frac{1}{|G|} \int_{G} u d x$ the projection onto $\mathcal{N}$ and define $P_{s} u:=\left(\mathcal{I}-P_{c}\right) u$ for the projection onto the orthogonal complement, where $\mathcal{I}$ is the identity operator on $\left[\mathcal{L}^{2}(G)\right]^{n}$. We define $\mathcal{L}_{n}^{p}:=$ $\left[\mathcal{L}^{p}(G)\right]^{n}$.

The operator $\mathcal{A}$ given by Definition 1 generates an analytic semigroup $\left\{e^{t \mathcal{A}}\right\}_{t \geq 0}$ (cf. Dan Henry [10] or Pazy [16]), on $\mathcal{L}_{n}^{p}$ for all $p \geq 2$. It has the following property: There is an $\omega>0$ such that for all $t>0$ and all $u \in \mathcal{L}_{n}^{p}$

$$
\left\|e^{t \mathcal{A}} P_{s} u\right\|_{\mathcal{L}_{n}^{p}} \leq M e^{-\omega t}\left\|P_{s} u\right\|_{\mathcal{L}_{n}^{p}}
$$


where $\omega$ depends in general on $d_{i}$.

Moreover, we obtain

$$
\left\|e^{t \mathcal{A}} u\right\|_{\mathcal{L}_{n}^{p}} \leq M\|u\|_{\mathcal{L}_{n}^{p}}
$$

Also, we suppose

Assumption 3 There is a constant $M>0$ such that for all $t>0$ and $u \in \mathcal{L}_{n}^{m p}$

$$
\left\|e^{t \mathcal{A}} u\right\|_{\mathcal{L}_{n}^{m p}} \leq M\left(1+t^{-\alpha}\right)\|u\|_{\mathcal{L}_{n}^{p}}
$$

with $\alpha=\frac{d}{p}\left(\frac{m-1}{m}\right) \in(0,1)$.

The previous assumption is needed for the existence of the solutions and global bounds. Equation (10) follows the Sobolev-embedding of $W^{\alpha, p}$ into $\mathcal{L}^{m p}$. The main assumption is that the coefficient is between 0 and 1 .

Immediate conclusion of Assumption 3 and Equation (8) is

$$
\left\|e^{t \mathcal{A}} P_{s} u\right\|_{\mathcal{L}_{n}^{m p}} \leq M\left(1+t^{-\alpha}\right) e^{-\omega t}\left\|P_{s} u\right\|_{\mathcal{L}_{n}^{p}},
$$

where for simplicity we denote different constants $\omega, M$ by the same name.

For the noise we suppose:

Assumption 4 Let $W=\left(W_{1}, \ldots ., W_{n}\right)$ be a collection of $n$ independent Wiener process on an abstract probability space $(\Omega, F, \mathbb{P})$ with a bounded covariance operator $Q_{i}: \mathcal{L}^{2}(\partial G) \rightarrow \mathcal{L}^{2}(\partial G)$ defined by $Q_{i} f_{k}=\alpha_{i, k} f_{k}$ for $i=1,2, . ., n$, where $\left(\alpha_{i, k}\right)_{k \in \mathbb{N}_{0}}$ is a bounded sequence of real numbers and $\left(f_{k}\right)_{k \in \mathbb{N}_{0}}$ be any orthonormal basis on $\mathcal{L}^{2}(\partial G)$ with $f_{0} \equiv$ Constant. For $t \geq 0$ we can write $W_{i}(t)$ (cf. Da Prato and Zabczyk [7]) as

$$
W_{i}(t)=\sum_{k \in \mathbb{N}_{0}} \alpha_{i, k} \beta_{i, k}(t) f_{k} \text { for } i=1,2, . ., n,
$$

where $\left(\beta_{i, k}\right)_{k \in \mathbb{N}_{0}}$ are independent, standard Brownian motions in $\mathbb{R}$. Also, we assume by using the orthonormal basis $g_{k}$ of $\mathcal{A}_{i}$ in $\mathcal{L}^{2}(G)$ that for some small $\gamma \in\left(0, \frac{1}{2}\right)$

$$
\sum_{k, \ell=1}^{\infty}\left(\lambda_{k}+\lambda_{\ell}\right)^{2 \gamma+2 \gamma_{1}-1} q_{k, \ell}^{i, i}<\infty \text { for } i=1,2, . ., n,
$$

where the covariance $q_{k, \ell}^{i, j}$ is defined by

$$
q_{j, k}^{i, \ell}=\frac{1}{t} \mathbb{E}\left(\tilde{W}_{i, j}(t) \tilde{W}_{\ell, k}(t)\right)= \begin{cases}0 & \text { if } i \neq \ell, \\ \left\langle Q_{i} g_{j}, g_{k}\right\rangle_{\mathcal{L}^{2}(\partial G)} & \text { if } i=\ell,\end{cases}
$$

with

$$
\tilde{W}_{i, j}=\left\langle W_{i}, g_{j}\right\rangle_{\mathcal{L}^{2}(\partial G)} .
$$

For the nonlinearity we assume

Assumption 5 The nonlinearity $\mathcal{F}$ is a polynomial of at most degree $m$. Thus for all $p \geq 1$ it is bounded by

$$
\|\mathcal{F}(u)\|_{\mathcal{L}_{n}^{p}} \leq C\left(1+\|u\|_{\mathcal{L}_{n}^{p m}}^{m}\right) \text { for all } u \in \mathcal{L}_{n}^{p m} .
$$

where $m=\max \left(m_{1}, \ldots . ., m_{n}\right)$ and the $m_{i}$ are the degrees of the polynomials $\mathcal{F}_{i}$. 
The following assumption ensures, that the noise is mass-conserving and that various series converge. This is used in Case 1 only.

Assumption 6 Assume for $i=1,2, . ., n$ that

$$
\alpha_{i, 0}=0,
$$

and for any $N \leq m$ and any $\ell \in\{1, \ldots, N\}^{n}$

$$
\sum_{k_{1}, k_{2}, . ., k_{N}=1}^{\infty}\left(\frac{1}{\sum_{i=1}^{N} d_{\ell_{i}} \lambda_{k_{i}}} \prod_{i=1}^{N} \lambda_{k_{i}}^{2 \gamma_{1}-1} \mathrm{q}_{k_{i}, k_{i}}^{\ell_{i}, \ell_{i}}\right)^{\frac{1}{2}}<\infty .
$$

Remark 7 Condition (17) for all $N \geq 1$, for example in case $\gamma_{1}=0$, is implied by the weaker condition

$$
\sum_{k=1}^{\infty} \frac{\left(\mathrm{q}_{k, k}^{i, i}\right)^{\frac{1}{2}}}{\lambda_{k}^{\frac{1}{2}+\frac{1}{2 m}}}<\infty .
$$

We fix a universal $T_{0}>0$ that is the upper bound for all times involved.

The following two assumptions are used in the two cases separately. They are usually lemmas that follows directly from the fact that $\mathcal{F}$ is a polynomial. Note that $T_{1}$ in general depends on the initial condition $b(0)$.

Assumption 8 Let $b(t)$ in $\mathcal{N}$ be the solution of (3). Suppose there is a stopping time $T_{1} \leq T_{0}$ and a constant $C>0$, such that

$$
\sup _{\left[0, T_{1}\right]}|b| \leq C .
$$

Assumption 9 Let $b(t)$ in $\mathcal{N}$ be the solution of (5). Suppose there is a stopping time $T_{1} \leq T_{0}$ and $C>0$, such that for sufficiently large $\zeta \gg 1$ and for $\delta>0$ and $\kappa \in\left(0, \frac{1}{m+1}\right)$

$$
\mathbb{P}\left(\sup _{t \in\left[0, T_{1}\right]}|b(t)|^{m-1} \leq C \ln \left(\varepsilon^{-\frac{1}{\zeta}}\right)\right) \geq 1-\varepsilon^{\delta \kappa} .
$$

We remark that $\zeta$ depends mainly on $T_{0}$ and $\kappa$ (cf. Section 8$)$.

For our result we rely on a cut off argument. We consider only solutions $u$ that are not too large, as given by the next definition.

Definition 10 For a mild solution $u$ of (1) we define for $\kappa \in\left(0, \frac{1}{m+1}\right)$ the stopping time $\tau^{*}$ as

$$
\tau^{*}:=T_{0} \wedge \inf \left\{t>0:\|u\|_{\mathcal{L}_{n}^{2 m}}>\varepsilon^{-\kappa}\right\} .
$$

We give error estimates in terms of the following $\mathcal{O}$-notation.

Definition 11 For a real-valued family of processes $\left\{X_{\varepsilon}(t)\right\}_{t>0}$ we say that $X_{\varepsilon}$ is of order $f_{\varepsilon}$, i.e. $X_{\varepsilon}=\mathcal{O}\left(f_{\varepsilon}\right)$, if for every $p \geq 1$ there exists a constant $C_{p}$ such that

$$
\mathbb{E} \sup _{t \in\left[0, \tau^{*}\right]}\left|X_{\varepsilon}(t)\right|^{p} \leq C_{p} f_{\varepsilon}^{p}
$$

We use also the analogous notation for time-independent random variables. 
Definition 12 (Multi-Index Notation) Let $\ell \in \mathbb{N}_{0}^{n}$, i.e. $\ell=\left(\ell_{1}, \ell_{2}, \ldots \ldots, \ell_{n}\right)$ be a vector of nonnegative integers, $u=\left(u_{1}, u_{2}, \ldots u_{n}\right)$. Then we define

$$
|\ell|=\sum_{i=1}^{n} \ell_{i}, \quad \ell !=\prod_{i=1}^{n} \ell_{1} !, \quad u^{\ell}=\prod_{i=1}^{n} u_{i}^{\ell_{i}}, \quad D^{\ell}=\partial_{u_{1}}^{\ell_{1}} \partial_{u_{2}}^{\ell_{2}} \ldots . . \partial_{u_{n}}^{\ell_{n}}
$$

\section{Random boundary conditions}

Definition 13 (Neumann map) The Neumann map $\mathcal{D}: \mathcal{L}^{2}(\partial G) \rightarrow \mathcal{H}^{\frac{3}{2}}(G)$ is a continuous linear operator. It is defined for $f \in \mathcal{L}^{2}(\partial G)$ as the solution $\mathcal{D} f$ of

$$
(1-\Delta) \mathcal{D} f=0 \quad \text { and } \quad \partial_{\nu}(\mathcal{D} f)=f .
$$

With a slight abuse of notation, we also denote by $\mathcal{D}$ the extension from $\mathcal{L}_{n}^{2}(\partial G)$ to $\left[\mathcal{H}^{\frac{3}{2}}(G)\right]^{n}$.

Definition 14 Define the stochastic convolution $\mathcal{Z}(t)$ as

$$
\mathcal{Z}(t)=\sigma_{\varepsilon}(1-\Delta) \int_{0}^{t} e^{\varepsilon^{-2}(t-s) \mathcal{A}} \mathcal{D} d W(s) .
$$

The next lemma expands the stochastic convolution $\mathcal{Z}$ as a Fourier series.

Lemma 15 Under Assumption 4 let $\mathcal{Z}$ be the stochastic convolution defined in (22), then (with $\tilde{W}_{i, j}$ defined in (15))

$$
\mathcal{Z}(t)=\sigma_{\varepsilon} \sum_{i=1}^{n} \sum_{j=0}^{\infty} \int_{0}^{t} e^{-\varepsilon^{-2} d_{i}(t-s) \lambda_{j}} d \tilde{W}_{i, j}(s) g_{j} \cdot e_{i} .
$$

Proof. Writing $\mathcal{Z}(t)$ in Fourier expansion yields

$$
\mathcal{Z}(t)=\sum_{i=1}^{n} \sum_{j=0}^{\infty}\left\langle\mathcal{Z}(t), e_{i} g_{j}\right\rangle g_{j} \cdot e_{i}
$$

Using Equation (22)

$$
\begin{aligned}
\left\langle\mathcal{Z}(t), e_{i} g_{j}\right\rangle_{\mathcal{L}_{n}^{2}(G)} & =\left\langle\mathcal{Z}_{i}(t), g_{j}\right\rangle_{\mathcal{L}^{2}(G)} \\
& =\left\langle\sigma_{\varepsilon}(1-\Delta) \int_{0}^{t} e^{\varepsilon^{-2}(t-s) d_{i} \Delta} \mathcal{D} d W_{i}(s), g_{j}\right\rangle_{\mathcal{L}^{2}(G)} \\
& =\sigma_{\varepsilon} \int_{0}^{t} e^{-\varepsilon^{-2} d_{i}(t-s) \lambda_{j}}\left\langle\mathcal{D} d W_{i}(s),(1-\Delta) g_{j}\right\rangle_{\mathcal{L}^{2}(G)} \\
& =\sigma_{\varepsilon} \int_{0}^{t} e^{-\varepsilon^{-2} d_{i}(t-s) \lambda_{j}}\left\{\left\langle\mathcal{D} d W_{i}(s), g_{j}\right\rangle_{\mathcal{L}^{2}(G)}-\left\langle\mathcal{D} d W(s), \Delta g_{j}\right\rangle_{\mathcal{L}^{2}(G)}\right\} \\
& =\sigma_{\varepsilon} \int_{0}^{t} e^{-\varepsilon^{-2} d_{i}(t-s) \lambda_{j}}\left\{\left\langle(1-\Delta) \mathcal{D} d W_{i}(s), g_{j}\right\rangle_{\mathcal{L}^{2}(G)}\right. \\
& \left.+\left\langle\partial_{\nu} \mathcal{D} d W_{i}(s), g_{j}\right\rangle_{\mathcal{L}_{n}^{2}(\partial G)}\right\} \\
& =\sigma_{\varepsilon} \int_{0}^{t} e^{-\varepsilon^{-2} d_{i}(t-s) \lambda_{j}}\left\langle d W_{i}(s), g_{j}\right\rangle_{\mathcal{L}^{2}(G)},
\end{aligned}
$$


where we used Gauss-Green formula and Definition 13 Hence

$$
\mathcal{Z}(t)=\sigma_{\varepsilon} \sum_{i=1}^{n} \sum_{j=0}^{\infty} \int_{0}^{t} e^{-\varepsilon^{-2} d_{i}(t-s) \lambda_{j}} d \tilde{W}_{i, j}(s) g_{j} \cdot e_{i} .
$$

It is easy to check, that this series converges in $\mathcal{L}_{n}^{2}$.

\section{Limiting equation and main theorem}

In this section we derive formally the limiting equation for (1) and we state without proof the main theorem of this paper. First, let us define the mild solution of Equation (11) according to [8, 9] as follows

Definition 16 For any fixed $\varepsilon>0$, we call a $\mathcal{L}_{n}^{p}$-valued stochastic process u a mild solution of (1) in $\mathcal{L}_{n}^{p}$ if for all $t>0$ up to a positive stopping time

$$
u(t)=e^{\varepsilon^{-2} t \mathcal{A}} u(0)+\int_{0}^{t} e^{\varepsilon^{-2}(t-s) \mathcal{A}} \mathcal{F}(u(s)) d s+\mathcal{Z}(t) .
$$

Because we are working with a locally Lipschitz nonlinearity, under Assumption 3. the existence and uniqueness of solutions is standard, once $\mathcal{Z}$ is sufficiently regular. See e.g. [7] and [9.

We can rewrite Equation (24) by using Equation (23) as

$$
\begin{aligned}
u(t)= & e^{\varepsilon^{-2} t \mathcal{A}} u(0)+\int_{0}^{t} e^{\varepsilon^{-2}(t-s) \mathcal{A}} \mathcal{F}(u(s)) d s \\
& +\sigma_{\varepsilon} \sum_{i=1}^{n} \sum_{j=0}^{\infty} \int_{0}^{t} e^{-\varepsilon^{-2} d_{i}(t-s) \lambda_{j}} d \tilde{W}_{i, j}(s) g_{j} \cdot e_{i},
\end{aligned}
$$

with $\tilde{W}_{i, j}$ defined in (15).

Now, let us discuss two cases depending on $\sigma_{\varepsilon}$ and $\alpha_{i, 0}$ for $i=1, \ldots, n$.

\subsection{First case: $\sigma_{\varepsilon}=\varepsilon^{-1}$ and $\alpha_{i, 0}=0$ for $i=1, . ., n$}

In this case Equation (25) takes the form

$$
u(t)=e^{\varepsilon^{-2} t \mathcal{A}} u(0)+\int_{0}^{t} e^{\varepsilon^{-2}(t-s) \mathcal{A}} \mathcal{F}(u(s)) d s+\mathcal{Z}^{s}(t),
$$

where

$$
\mathcal{Z}^{s}(t)=\sum_{i=1}^{n} \mathcal{Z}_{i}(t) e_{i}:=\sum_{i=1}^{n} \sum_{j=1}^{\infty} \mathcal{Z}_{i, j}(t) g_{j} \cdot e_{i},
$$

with

$$
\mathcal{Z}_{i}(t)=\sum_{j=1}^{\infty} \mathcal{Z}_{i, j}(t) g_{j} \text { for } i=1,2 \ldots ., n
$$

and

$$
\mathcal{Z}_{i, j}(t)=\varepsilon^{-1} \int_{0}^{t} e^{-\varepsilon^{-2} d_{i}(t-s) \lambda_{j}} d \tilde{W}_{i, j}(s) .
$$


In order to derive the limiting equation, we split the solution $u$ into

$$
u(t, x)=a(t)+\psi(t, x),
$$

with $a \in \mathcal{N}$ and $\psi \in \mathcal{S}$. Plugging (30) into (26) and projecting everything onto $\mathcal{N}$ and $\mathcal{S}$ we obtain (with $\mathcal{F}^{c}=P_{c} \mathcal{F}$ and $\mathcal{F}^{s}=P_{s} \mathcal{F}$ )

$$
a(t)=a(0)+\int_{0}^{t} \mathcal{F}^{c}(a+\psi) d s,
$$

and

$$
\psi(t)=e^{\varepsilon^{-2} t \mathcal{A}} \psi(0)+\int_{0}^{t} e^{\varepsilon^{-2}(t-\tau) \mathcal{A}_{s}} \mathcal{F}^{s}(a+\psi) d \tau+\mathcal{Z}^{s}(t) .
$$

Formally, we see later (cf. Lemma 22) that $\psi$ is well approximated by the fast Ornstein-Uhlenbeck process $\mathcal{Z}^{s}$. Thus, we can eliminate $\psi$ in (31) by explicitly averaging over the fast modes.

Now the first main result of this paper is:

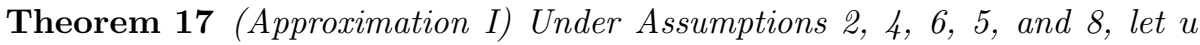
be a solution of (1) with splitting $u=a+\psi$ defined in (30) with the initial condition $u(0)=a(0)+\psi(0)$ with $a(0) \in \mathcal{N}$ and $\psi(0) \in S$ where $a(0)$ and $\psi(0)$ are of order one, and $b$ is a solution of (3) with $b(0)=a(0)$. Then for all $p>0$ and all $\kappa \in\left(0, \frac{1}{2 m+1}\right)$, there exist a constant $C>0$ such that

$$
\mathbb{P}\left(\sup _{t \in\left[0, T_{1} \wedge \tau^{*}\right]}\|u(t)-b(t)-\mathcal{Q}(t)\|_{\mathcal{L}_{n}^{p}}>\varepsilon^{1-2 m \kappa-\kappa}\right) \leq C \varepsilon^{p},
$$

where with fast $O U$-process $\mathcal{Z}^{s}$ defined in (27)

$$
\mathcal{Q}(t)=e^{\varepsilon^{-2} t \mathcal{A}_{s}} \psi(0)+\mathcal{Z}^{s}(t) .
$$

We see that the first part of (34) depending on the initial condition decays exponentially fast on the time-scale of order $\mathcal{O}\left(\varepsilon^{2}\right)$.

Corollary 18 If in the previous theorem we additionally assume that Assumption 3 holds and $\|\psi(0)\|_{\mathcal{L}_{n}^{m p}} \leq C$ for some $C>0$, then we can replace $T_{1} \wedge \tau^{*}$ in (33) by $T_{1}$.

Remark 19 In case of Corollary 18 we can bound the error even in $\mathcal{L}_{n}^{p m}$.

\subsection{Second case $\sigma_{\varepsilon}=1$ and $\alpha_{i, 0} \neq 0$ for $i=1, . ., n$}

In this case (25) takes the form

$$
\begin{aligned}
u(t)= & e^{\varepsilon^{-2} t \mathcal{A}} u(0)+\int_{0}^{t} e^{\varepsilon^{-2}(t-s) \mathcal{A}} \mathcal{F}(u(s)) d s \\
& +\sum_{i=1}^{n} \sum_{j=0}^{\infty} \int_{0}^{t} e^{-\varepsilon^{-2} d_{i}(t-s) \lambda_{j}} d \tilde{W}_{i, j}(s) g_{j} \cdot e_{i} .
\end{aligned}
$$

Again (cf. (30)) we split the solution $u$ into $u(t, x)=a(t)+\varepsilon \psi(t, x)$. Plugging (30) into (35) and projecting everything onto $\mathcal{N}$ and $\mathcal{S}$ yields

$$
a(t)=a(0)+\int_{0}^{t} \mathcal{F}^{c}(a+\varepsilon \psi) d s+\sum_{i=1}^{n} \tilde{W}_{i, 0}(t) g_{0} \cdot e_{i},
$$


and

$$
\psi(t)=e^{\varepsilon^{-2} t \mathcal{A}} \psi(0)+\frac{1}{\varepsilon} \int_{0}^{t} e^{\varepsilon^{-2}(t-\tau) \mathcal{A}_{s}} \mathcal{F}^{s}(a+\varepsilon \psi) d \tau+\mathcal{Z}^{s}(t),
$$

where $\mathcal{Z}^{s}(t)$ was defined in (27). We write (36) as

$$
a_{i}(t)=a_{i}(0)+\int_{0}^{t} \mathcal{F}_{i}^{c}(a+\varepsilon \psi) d s+\tilde{W}_{i, 0}(t) g_{0} \text { for } i=1,2 \ldots, n .
$$

Now, applying Taylor's expansion to the function $\mathcal{F}_{i}^{c}: \mathcal{L}^{2}(G) \rightarrow \mathbb{R}$, yields the following stochastic limiting equation with error

$$
a_{i}(t)=a_{i}(0)+\int_{0}^{t} \mathcal{F}_{i}(a) d s+\tilde{W}_{i, 0}(t) g_{0}+R_{i}^{(2)}(t),
$$

where

$$
R_{i}^{(2)}(t)=\sum_{|\ell| \geq 1} P_{c} \int_{0}^{t} \frac{D^{\ell} \mathcal{F}_{i}(a)}{\ell !}(\varepsilon \psi)^{\ell} d \tau=\mathcal{O}\left(\varepsilon^{1-}\right) .
$$

The second main result of this paper is:

Theorem 20 (Approximation II) Under Assumptions 2, 4, 5 and 9, let $u$ be a solution of (1) with splitting $u=a+\varepsilon \psi$ defined in (30) with the initial condition $u(0)=a(0)+\varepsilon \psi(0)$ with $a(0) \in \mathcal{N}$ and $\psi(0) \in S$ where $a(0)$ and $\psi(0)$ are of order one, and $b$ is a solution of (5) with $b(0)=a(0)$. Then for all $p>0$, for sufficiently large $\zeta \gg 1$ and all $\kappa \in\left(0, \frac{1}{m+2}\right)$, there exists $C>0$ such that

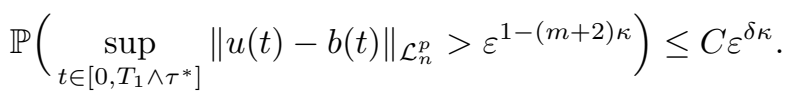

In our examples if we assume $\mathbb{E} \exp \left\{c \delta|b(0)|^{m-1}\right\} \leq C$ for some suitable $c>0$ and for one $\delta>0$, then Assumption 9 is true. See Section 8.1.

Corollary 21 If in the previous theorem additionally Assumption 3 holds and $\|\psi(0)\|_{\mathcal{L}_{n}^{m p}} \leq C$ for $C>0$, then we can replace $T_{1} \wedge \tau^{*}$ in (40) by $T_{1}$.

The sufficiently large $\zeta$ depends mainly on $\kappa$ and $T_{0}$.

\section{Bounds for the high modes}

Let us summarize Equations (32) and (37) for $\rho \in\{0,1\}$ by

$$
\psi(t)=e^{\varepsilon^{-2} t \mathcal{A}} \psi(0)+\varepsilon^{-\rho} \int_{0}^{t} e^{\varepsilon^{-2}(t-\tau) \mathcal{A}_{s}} \mathcal{F}^{s}(a+\varepsilon \psi) d \tau+\mathcal{Z}^{s}(t) .
$$

In the first lemma of this section, we see that $\psi$ is well approximated by the fast Ornstein-Uhlenbeck process $\mathcal{Z}^{s}$ (cf. (27)).

Lemma 22 Under Assumption [5, there is a constant $C>0$ such that for $p \geq 1$ and $\kappa>0$ from the definition of $\tau^{*}$

$$
\mathbb{E} \sup _{t \in\left[0, \tau^{*}\right]}\left\|\psi(t)-e^{\varepsilon^{-2} t \mathcal{A}} \psi(0)-\mathcal{Z}^{s}(t)\right\|_{\mathcal{L}_{n}^{p}}^{p} \leq C \varepsilon^{2 p-p \rho-m p \kappa} .
$$


Proof. From (41) using semigroup estimates and Assumption [5 we obtain

$$
\begin{aligned}
\left\|\psi(t)-e^{\varepsilon^{-2} t \mathcal{A}} \psi(0)-\mathcal{Z}^{s}(t)\right\|_{\mathcal{L}_{n}^{p}} & =\frac{1}{\varepsilon^{\rho}}\left\|\int_{0}^{t} e^{\varepsilon^{-2} \mathcal{A}_{s}(T-\tau)} \mathcal{F}^{s}(u) d \tau\right\|_{\mathcal{L}_{n}^{p}} \\
& \leq C \varepsilon^{-\rho} \sup _{\tau \in\left[0, \tau^{*}\right]}\left\|\mathcal{F}^{s}(u)\right\|_{\mathcal{L}_{n}^{p}} \int_{0}^{t} e^{-\varepsilon^{-2} \omega(t-\tau)} d \tau \\
& \leq C \varepsilon^{2-\rho} \sup _{\tau \in\left[0, \tau^{*}\right]}\left(1+\|u\|_{\mathcal{L}_{n}^{p m}}^{m}\right) \leq C \varepsilon^{2-\rho-m \kappa} .
\end{aligned}
$$

Lemma 23 Under Assumptions 2 and 因 for every $\kappa_{0}>0$ and $p \geq 1$ there is a constant $C$, depending on $p, \alpha_{k}, \lambda_{k}, \kappa_{0}$ and $T_{0}$, such that

$$
\mathbb{E} \sup _{t \in\left[0, T_{0}\right]}\left\|\mathcal{Z}^{s}(t)\right\|_{\mathcal{L}_{n}^{p}}^{p} \leq C \varepsilon^{-\kappa_{0}},
$$

where $\mathcal{Z}^{s}(t)$ was defined in (27).

Proof. We use the celebrated factorization method introduced in [7] to prove the bound on $\mathcal{Z}^{s}(t)=\sum_{i=1}^{n} \mathcal{Z}_{i}(t) e_{i}$, which is based on the following elementary identity

$$
\int_{\sigma}^{t}(t-r)^{\gamma-1}(r-\sigma)^{-\gamma} d r=\frac{\pi}{\sin (\pi \gamma)} \text { for } 0 \leq r \leq t, 0<\gamma<1 .
$$

Fix $\gamma \in\left(0, \frac{1}{2}\right)$. To prove (43), it is enough to bound $\mathcal{Z}_{i}$ for $i=1, \ldots n$. We obtain from Equation (28) that

$$
\mathcal{Z}_{i}(t)=\sum_{j=1}^{\infty} \varepsilon^{-1} \int_{0}^{t} e^{-\varepsilon^{-2} d_{i}(t-s) \lambda_{j}} d \tilde{W}_{i, j}(s) g_{j}=\varepsilon^{-1} \int_{0}^{t} e^{\varepsilon^{-2}(t-s) \mathcal{A}_{i}} d \tilde{W}_{i}(s),
$$

where

$$
\tilde{W}_{i}(t)=\sum_{j=1}^{\infty} \tilde{W}_{i, j}(s) g_{j} \text { for } i=1,2, \ldots, n .
$$

Using Identity (44) with Equation (45), we obtain:

$$
\mathcal{Z}_{i}(t)=C_{\gamma} \varepsilon^{-1} \int_{0}^{t} e^{\varepsilon^{-2}(t-\sigma) \mathcal{A}_{i}}\left[\int_{\sigma}^{t}(t-s)^{\gamma-1}(s-\sigma)^{-\gamma} d r\right] d \tilde{W}_{i}(\sigma) .
$$

From the stochastic Fubini theorem, we obtain

$$
\mathcal{Z}_{i}(t)=C_{\gamma} \varepsilon^{-1} \int_{0}^{t} e^{\varepsilon^{-2}(t-s) \mathcal{A}_{i}}(t-s)^{\gamma-1} y_{i}(s) d s,
$$

where

$$
\begin{aligned}
y_{i}(s) & =\int_{0}^{s} e^{\varepsilon^{-2}(s-\sigma) \mathcal{A}_{i}}(s-\sigma)^{-\gamma} d \tilde{W}_{i}(\sigma) \\
& =\sum_{j=1}^{\infty} \int_{0}^{s} e^{-\varepsilon^{-2} d_{i}(s-\sigma) \lambda_{j}}(s-\sigma)^{-\gamma} d \tilde{W}_{i, j}(\sigma) g_{j} .
\end{aligned}
$$


Taking $\|\cdot\|_{\mathcal{L}_{n}^{p}}^{p}$ on both sides of (46) and using (묘), we obtain

$$
\left\|\mathcal{Z}_{i}(t)\right\|_{\mathcal{L}^{p}}^{p} \leq C_{\gamma}^{p} \varepsilon^{-p}\left(\int_{0}^{t} e^{-\varepsilon^{-2}(t-s) \omega}(t-s)^{\gamma-1}\left\|y_{i}(s)\right\|_{\mathcal{L}^{p}} d s\right)^{p} .
$$

Using Hölder inequality with $\frac{1}{p}+\frac{1}{q}=1$ for sufficiently large $p$ implies

$$
\begin{aligned}
\left\|\mathcal{Z}_{i}(t)\right\|_{\mathcal{L}^{p}}^{p} & \leq C_{\gamma}^{p} \varepsilon^{-p}\left(\int_{0}^{t} e^{-\varepsilon^{-2}(t-s) \omega}(t-s)^{q \gamma-q} d s\right)^{\frac{p}{q}} \cdot \int_{0}^{t}\left\|y_{i}(s)\right\|_{\mathcal{L}^{p}}^{p} d s \\
& \leq C \varepsilon^{-2+2 p\left(\gamma-\frac{1}{2}\right)} \int_{0}^{t}\left\|y_{i}(s)\right\|_{\mathcal{L}^{p}}^{p} d s .
\end{aligned}
$$

Taking supremum after expectation, yields

$$
\mathbb{E} \sup _{t \in\left[0, T_{0}\right]}\left\|\mathcal{Z}_{i}(t)\right\|_{\mathcal{L}^{p}}^{p} \leq C \varepsilon^{-2+2 p\left(\gamma-\frac{1}{2}\right)} \cdot \int_{0}^{T_{0}} \mathbb{E}\left\|y_{i}(s)\right\|_{\mathcal{L}^{p}}^{p} d s .
$$

Now, we bound $\mathbb{E}\left\|y_{i}(s)\right\|_{\mathcal{L}^{p}}^{p}$. By Gaussianity

$$
\mathbb{E}\left\|y_{i}(s)\right\|_{\mathcal{L}^{p}}^{p}=\mathbb{E} \int_{D}\left|y_{i}(s, x)\right|^{p} d x \leq C_{p}\left(\int_{D} \mathbb{E}\left|y_{i}(s, x)\right|^{2}\right)^{\frac{p}{2}} d x .
$$

Hence by Definition of $y_{i}$ (47)

$$
\begin{aligned}
\mathbb{E}\left|y_{i}(s, x)\right|^{2} & =\mathbb{E}\left|\sum_{j=1}^{\infty} \int_{0}^{s} e^{-\varepsilon^{-2} d_{i}(s-\sigma) \lambda_{j}}(s-\sigma)^{-\gamma} d \tilde{W}_{i, j}(\sigma) g_{j}(x)\right|^{2} \\
& =C \sum_{j, k=1}^{\infty} q_{j, k}^{i, i} \int_{0}^{s} e^{-\varepsilon^{-2} d_{i}(s-\sigma)\left(\lambda_{j}+\lambda_{k}\right)}(s-\sigma)^{-2 \gamma} d \sigma g_{j}(x) g_{k}(x),
\end{aligned}
$$

where we used the definition of covariance operator (14). Hence, using the bounds on $g_{j}$

$$
\mathbb{E}\left|y_{i}(s)\right|^{2} \leq C \varepsilon^{2-4 \gamma} \sum_{j, k=1}^{\infty}\left(\lambda_{j}+\lambda_{k}\right)^{2 \gamma+2 \gamma_{1}-1} q_{j, k}^{i, i} \leq C \varepsilon^{2-4 \gamma},
$$

where we used (13). Thus

$$
\sup _{t \in\left[0, T_{0}\right]} \mathbb{E}\left\|y_{i}(s)\right\|_{\mathcal{L}^{p}}^{p} \leq C \varepsilon^{p-2 p \gamma} .
$$

Now, returning to Equation (48) and using Equation (50), yields

$$
\mathbb{E} \sup _{t \in\left[0, T_{0}\right]}\left\|\mathcal{Z}_{i}(t)\right\|_{\mathcal{L}_{n}^{p}}^{p} \leq C \varepsilon^{-2} .
$$

We finish the proof by using Hölder inequality to derive for all $p>1$ and sufficiently large $q>\frac{2}{\kappa_{0}}$

$$
\mathbb{E} \sup _{t \in\left[0, T_{0}\right]}\left\|\mathcal{Z}_{i}(t)\right\|_{\mathcal{L}_{n}^{p}}^{p} \leq\left(\mathbb{E} \sup _{t \in\left[0, T_{0}\right]}\left\|\mathcal{Z}_{i}(t)\right\|_{\mathcal{L}_{n}^{p}}^{p q}\right)^{\frac{1}{q}} \leq C \varepsilon^{-\kappa_{0}} .
$$

The following corollary states that $\psi(t)$ is with high probability much smaller than $\varepsilon^{-\kappa}$ as assumed the Definition 10 for $t \leq \tau^{*}$. We show later $\tau^{*} \geq T_{0}$ with high probability. 
Corollary 24 Under the assumptions of Lemmas 220 and 23], if $\psi(0)=\mathcal{O}(1)$, then for $p>0$ and $\rho=0$ or 1 there exist a constant $C>0$ such that for $\kappa_{0} \leq \kappa$

$$
\mathbb{E} \sup _{t \in\left[0, \tau^{*}\right]}\|\psi(t)\|_{\mathcal{L}_{n}^{p}}^{p} \leq C \varepsilon^{-\kappa_{0}} .
$$

Proof. By triangle inequality and Lemma 23, we obtain from (42)

$$
\mathbb{E} \sup _{t \in\left[0, \tau^{*}\right]}\|\psi(t)\|_{\mathcal{L}_{n}^{p}}^{p} \leq C+C \varepsilon^{2 p-p \rho-m p \kappa}+C \varepsilon^{-\kappa_{0}}
$$

which implies (51) for $\kappa<\frac{2-\rho}{m}$.

Let us now state a result similar to averaging. When we integrate over the fast decaying contribution of the initial condition in $\psi$, then this leads to terms of order $\mathcal{O}\left(\varepsilon^{2}\right)$.

Lemma 25 For $q \geq 1$ there exists a constant $C>0$ such that

$$
\int_{0}^{t}\left\|e^{\tau \varepsilon^{-2} \mathcal{A}_{s}} \psi(0)\right\|_{\mathcal{L}_{n}^{p}}^{q} d \tau \leq C \varepsilon^{2}\|\psi(0)\|_{\mathcal{L}_{n}^{p}}^{q} \text { for } \psi(0) \in \mathcal{L}_{n}^{p} .
$$

Proof. Using (8) we obtain

$$
\int_{0}^{t}\left\|e^{\varepsilon^{-2} \mathcal{A}_{s} \tau} \psi(0)\right\|_{\mathcal{L}_{n}^{p}}^{q} d \tau \leq c \int_{0}^{T} e^{-q \varepsilon^{-2} \omega \tau}\|\psi(0)\|_{\mathcal{L}_{n}^{p}}^{q} d \tau \leq \frac{\varepsilon^{2}}{q \omega}\|\psi(0)\|_{\mathcal{L}_{n}^{p}}^{q} .
$$

\section{Averaging over the fast OU-process}

Lemma 26 Let Assumption 4 hold and consider $\mathcal{Z}_{i, j}(t)$ as defined in (29). Then for arbitrary $\delta_{0} \in\left(0, \frac{1}{2}\right)$ we obtain

$$
\mathcal{Z}_{i, j}(t)=\lambda_{j}^{-\frac{1}{2}\left(1-\delta_{0}\right)}\left(\mathrm{q}_{j, j}^{i, i}\right)^{\frac{1}{2}} \mathcal{O}\left(\varepsilon^{-\delta_{0}}\right),
$$

and

$$
\mathcal{Z}_{i, j}(t) \mathcal{Z}_{\ell, k}(t)=\left(\lambda_{j} \lambda_{k}\right)^{-\frac{1}{2}\left(1-\delta_{0}\right)}\left(\mathrm{q}_{j, j}^{i, i} \mathrm{q}_{k, k}^{\ell, \ell}\right)^{\frac{1}{2}} \mathcal{O}\left(\varepsilon^{-2 \delta_{0}}\right) .
$$

Moreover, the $\mathcal{O}$-terms are uniform in $i, j, k$ and $\ell$.

Proof. For the first part, we follow the same steps as in Lemma 23 to obtain

$$
\mathbb{E} \sup _{t \in\left[0, T_{0}\right]}\left|\mathcal{Z}_{i, j}(t)\right|^{p} \leq C \varepsilon^{-2}\left(\lambda_{j}\right)^{1-\frac{1}{2} p}\left(q_{j, j}^{i, i}\right)^{\frac{p}{2}} .
$$

Using Hölder inequality, we derive for sufficiently large $q$ and for a constant independent on $i$ and $j$

$$
\left(\mathbb{E} \sup _{t \in\left[0, T_{0}\right]}\left|\mathcal{Z}_{i, j}(t)\right|^{p}\right)^{1 / p} \leq C \lambda_{j}^{-\frac{1}{2}}\left(\varepsilon^{-2} \lambda_{j}\right)^{\frac{1}{p q}}\left(q_{j, j}^{i, i}\right)^{\frac{1}{2}} .
$$

We finish the proof by fixing $\delta_{0}=\frac{2}{p q}<\frac{1}{2}$ for large $q$ and $p$. 
For the second part we use Cauchy-Schwarz inequality to obtain

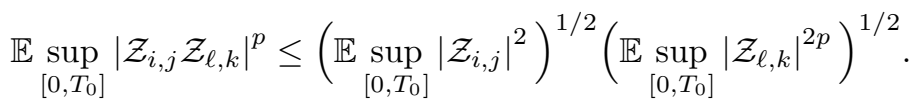

Using the first part, yields (53). $\quad \square$ In next corollary we state without proof the general case of Lemma 26. For the proof we can follow the same steps as in the proof of Lemma 26.

Corollary 27 Under the assumptions of Lemma 26] we have

$$
\prod_{j=1}^{N} \mathcal{Z}_{\ell_{j}, k_{j}}=\left(\prod_{j=1}^{N} \lambda_{k_{j}}\right)^{-\frac{1}{2}\left(1-\delta_{0}\right)}\left(\prod_{j=1}^{N} q_{k_{j}, k_{j}}^{\ell_{j}, \ell_{j}}\right)^{\frac{1}{2}} \mathcal{O}\left(\varepsilon^{-N \delta_{0}}\right) .
$$

Lemma 28 Let the assumptions of Lemma 26 hold and let $X$ be a real valued stochastic process such that for some small $r \geq 0$ we have $X(0)=\mathcal{O}\left(\varepsilon^{-r}\right)$. If $d X=G d T$ with $G=\mathcal{O}\left(\varepsilon^{-r}\right)$, then

$$
\begin{gathered}
\sup _{t \geq 0} \mathbb{E}\left|\mathcal{Z}_{i, j}(t)\right|^{2} \leq \frac{\mathrm{q}_{j, j}^{i, i}}{2 d_{i} \lambda_{j}} \\
\int_{0}^{t} X \mathcal{Z}_{i, j} d \tilde{W}_{k, m}=\left(\frac{\mathrm{q}_{m, m}^{k, k} \mathrm{q}_{j, j}^{i, i}}{\lambda_{j}}\right)^{\frac{1}{2}} \mathcal{O}\left(\varepsilon^{-r}\right),
\end{gathered}
$$

and

$$
\int_{0}^{t} X \prod_{\substack{j=1, j \neq i}}^{N} \mathcal{Z}_{\ell_{j}, k_{j}} d \tilde{W}_{\ell_{i}, k_{i}}=\left(\prod_{\substack{j=1, j \neq i}}^{N} \lambda_{k_{j}}\right)^{-\frac{1}{2}}\left(\prod_{j=1}^{N} \mathrm{q}_{k_{j}, k_{j}}^{\ell_{j}, \ell_{j}}\right)^{\frac{1}{2}} \mathcal{O}\left(\varepsilon^{-r}\right)
$$

Again all $\mathcal{O}$-terms are uniform in the indices $\ell_{j}$ and $k_{j}$.

Proof. For the first part, we use Itô isometry to obtain

$$
\mathbb{E}\left|\mathcal{Z}_{i, j}\right|^{2}=\frac{1}{\varepsilon^{2}} \mathbb{E}\left|\int_{0}^{t} e^{-\varepsilon^{-2} d_{i}(t-s) \lambda_{j}} d \tilde{W}_{i, j}\right|^{2}=\frac{q_{j, j}^{i, i}}{\varepsilon^{2}} \int_{0}^{t} e^{-2 \varepsilon^{-2} d_{i}(t-s) \lambda_{j}} d s \leq \frac{q_{j, j}^{i, i}}{2 d_{i} \lambda_{j}} .
$$

For the second part using Burkholder-Davis-Gundy theorem and Hölder inequality, yields

$$
\begin{aligned}
\mathbb{E} \sup _{t \in\left[0, T_{0}\right]}\left|\int_{0}^{t} X \mathcal{Z}_{i, j} d \tilde{W}_{k, m}\right|^{p} & \leq C_{p}\left(q_{m, m}^{k, k}\right)^{\frac{p}{2}} \mathbb{E}\left(\int_{0}^{T_{0}}|X|^{2}\left|\mathcal{Z}_{i, j}\right|^{2} d \sigma\right)^{\frac{p}{2}} \\
& \leq C_{p, T_{0}} \varepsilon^{-p r}\left(q_{m, m}^{k, k}\right)^{\frac{p}{2}} \int_{0}^{T_{0}} \mathbb{E}\left|\mathcal{Z}_{i, j}\right|^{p} d \sigma
\end{aligned}
$$

By Gaussianity and the first part we obtain

$$
\mathbb{E} \sup _{t \in\left[0, T_{0}\right]}\left|\int_{0}^{t} X \mathcal{Z}_{i, j} d \tilde{W}_{k, m}\right|^{p} \leq C_{p, T_{0}} \varepsilon^{-p r}\left(\frac{q_{m, m}^{k, k} q_{j, j}^{i, i}}{\lambda_{j}}\right)^{\frac{p}{2}} .
$$

Analogously, for the last term

$\mathbb{E} \sup _{t \in\left[0, T_{0}\right]}\left|\int_{0}^{t} X \prod_{\substack{j=1, j \neq i}}^{N} \mathcal{Z}_{\ell_{j}, k_{j}} d \tilde{W}_{\ell_{i}, k_{i}}\right|^{p} \leq C_{p, T_{0}}\left(q_{k_{i}, k_{i}}^{\ell_{i}, \ell_{i}}\right)^{\frac{p}{2}} \mathbb{E} \int_{0}^{T_{0}}|X|^{p} \prod_{\substack{j=1, j \neq i}}^{N}\left|\mathcal{Z}_{\ell_{j}, k_{j}}\right|^{p} d \sigma$.

Using Hölder, Gaussianity and the first part, we obtain (57).

In the following we state and prove the averaging lemma over the fast OUprocess $\mathcal{Z}_{i, j}$ (cf. (29) ). 


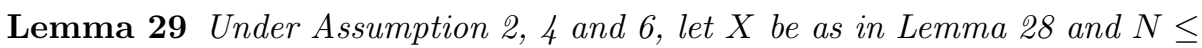
$m$. Then for $N$ odd

$$
\int_{0}^{t} X \prod_{i=1}^{N} \mathcal{Z}_{\ell_{i}, k_{i}} d s=A_{k_{1}, \cdots, k_{N}}^{\ell_{1}, \cdots \ell_{N}} \mathcal{O}\left(\varepsilon^{1-r}\right)
$$

and for $N$ even

$$
\begin{aligned}
\int_{0}^{t} X \prod_{i=1}^{N} \mathcal{Z}_{\ell_{i}, k_{i}} d s= & \frac{1}{2^{\frac{N}{2}}} \sum_{j \in \operatorname{Per}(N)} \prod_{\eta=1}^{N / 2} \frac{\mathrm{q}_{k_{j_{2 \eta-1}}, k_{j_{2 \eta}}}^{\ell_{j_{2}}, \ell_{j_{2}}}}{d_{\ell_{j_{2 \eta-1}}} \lambda_{k_{j_{2 \eta-1}}}+d_{\ell_{j_{2 \eta}}} \lambda_{k_{j_{2}}}} \int_{0}^{t} X d s \\
& +A_{k_{1}, \cdots, k_{N}}^{\ell_{1}, \cdots \ell_{N}} \mathcal{O}\left(\varepsilon^{1-r}\right),
\end{aligned}
$$

with

$$
\sum_{k_{1}=1}^{\infty} \cdots \sum_{k_{N}=1}^{\infty} A_{k_{1}, \cdots, k_{N}}^{\ell_{1}, \cdots, \ell_{N}} \prod_{i=1}^{N} \lambda_{k_{i}}^{\gamma_{1}}<\infty
$$

The $\mathcal{O}$-terms are again uniform in all indices.

We used $j \in \operatorname{Per}(N)$ if $j=\left(j_{1}, \ldots, j_{N}\right)$ is a permutation of $\{1, \ldots, N\}$.

Remark 30 The term

$$
\sum_{j \in \operatorname{Per}(N)} \prod_{\eta=1}^{N / 2} \frac{\mathrm{q}_{k_{j_{2 \eta-1}}, k_{j_{2 \eta}}}^{\ell_{j_{2 \eta-1}} \ell_{j_{2 \eta}}}}{\mathrm{d}_{\ell_{j_{2 \eta-1}}} \lambda_{k_{j_{2 \eta-1}}}+d_{\ell_{j_{2 \eta}}} \lambda_{k_{j_{2 \eta}}}}
$$

is summable over $k_{1}, \cdots, k_{N}$ by Condition (13).

Let us state explicitly some $A$ 's appearing in the proof of the theorem.

Example 31 For $N=1$ we have $A_{k}^{\ell}=\frac{1}{\lambda_{k}}\left(\mathrm{q}_{k, k}^{\ell, \ell}\right)^{\frac{1}{2}}$, and for $N=2$

$$
A_{k_{1}, k_{2}}^{\ell_{1}, \ell_{2}}=\left(\sum_{i=1}^{2} d_{\ell_{i}} \lambda_{k_{i}}\right)^{-1 / 2}\left(\prod_{i=1}^{2} \lambda_{k_{i}}^{-1} q_{k_{i}, k_{i}}^{\ell_{i}, \ell_{i}}\right)^{1 / 2}
$$

and for $N=3$

$$
A_{k_{1}, k_{2}, k_{3}}^{\ell_{1}, \ell_{2}, \ell_{3}}=\left(\frac{1}{\sum_{i=1}^{3} d_{\ell_{i}} \lambda_{k_{i}}} \prod_{i=1}^{3} \lambda_{k_{i}}^{-1} \mathrm{q}_{k_{i}, k_{i}, \ell_{i}}^{\ell_{i}}\right)^{\frac{1}{2}}+\sum_{\substack{j_{1}, j_{2}=1 \\ j_{1} \neq j_{2}}}^{3} \frac{\mathrm{q}_{k_{j_{1}}, k_{j_{2}}}^{\ell_{j_{1}}, \ell_{j_{2}}}}{d_{\ell_{j_{1}}} \lambda_{k_{j_{1}}}+d_{\ell_{j_{2}}} \lambda_{k_{j_{2}}}} \frac{\left(\mathrm{q}_{j, j}^{i, i}\right)^{\frac{1}{2}}}{\lambda_{j}} .
$$

For larger $N$ the terms have similar structure, but there are about $N / 2$ many.

Proof. Fix a small $\delta_{0}<\frac{1}{N}$ for $N>1$. First, recall $|X|=\mathcal{O}\left(\varepsilon^{-r}\right)$. For the first part we treat $N=1$ and 3 . The general case follows by induction.

For $N=1$ we apply Itô formula to the term $X \mathcal{Z}_{i, j}$ to obtain

$$
\int_{0}^{t} X \mathcal{Z}_{i, j} d s=-\frac{\varepsilon^{2}}{d_{i} \lambda_{j}} X(t) \mathcal{Z}_{i, j}(t)+\frac{\varepsilon^{2}}{d_{i} \lambda_{j}} \int_{0}^{t} G \mathcal{Z}_{i, j} d s+\frac{\varepsilon}{d_{i} \lambda_{j}} \int_{0}^{t} X d \tilde{W}_{i, j} .
$$


Using Lemmas 26] and Burkholder-Davis-Gundy theorem, yields

$$
\begin{aligned}
\int_{0}^{t} X \mathcal{Z}_{i, j} d s & =\left(q_{j, j}^{i, i}\right)^{\frac{1}{2}}\left[\frac{1}{\left(d_{i} \lambda_{j}\right) \lambda_{j}^{\frac{1}{2}-\frac{1}{2} \delta_{0}}} \mathcal{O}\left(\varepsilon^{2-r-\delta_{0}}\right)+\frac{1}{d_{i} \lambda_{j}} \mathcal{O}\left(\varepsilon^{1-r}\right)\right] \\
& =\frac{1}{\lambda_{j}}\left(q_{j, j}^{i, i}\right)^{\frac{1}{2}} \mathcal{O}\left(\varepsilon^{1-r}\right) .
\end{aligned}
$$

For $N \in\{3,5, .$.$\} we apply Itô formula to the term X \prod_{i=1}^{N} \mathcal{Z}_{\ell_{i}, k_{i}}$ to obtain

$$
\begin{aligned}
\int_{0}^{t} X \prod_{i=1}^{N} \mathcal{Z}_{\ell_{i}, k_{i}} d s= & \frac{1}{\sum_{i=1}^{N} d_{\ell_{i}} \lambda_{k_{i}}}\left\{\varepsilon^{2} X \prod_{i=1}^{N} \mathcal{Z}_{\ell_{i}, k_{i}}+\varepsilon^{2} \int_{0}^{t} G \prod_{i=1}^{N} \mathcal{Z}_{\ell_{i}, k_{i}} d s\right. \\
& +\varepsilon \sum_{j=1}^{N} \int_{0}^{t} X \prod_{\substack{i=1, i \neq j}}^{N} \mathcal{Z}_{\ell_{i}, k_{i}} d \tilde{W}_{\ell_{j}, k_{j}} \\
& \left.+\sum_{j_{1} \neq j_{2}=1}^{N} \int_{0}^{t} X \prod_{\substack{i=1, i \notin\left\{j_{1}, j_{2}\right\}}}^{N} \mathcal{Z}_{\ell_{i}, k_{i}} d \tilde{W}_{\ell_{j_{1}}, k_{j_{1}}} d \tilde{W}_{\ell_{j_{2}}, k_{j_{2}}}\right\}
\end{aligned}
$$

Using Corollary 27 and Lemma 28 to obtain

$$
\begin{aligned}
\int_{0}^{t} X \prod_{i=1}^{N} \mathcal{Z}_{\ell_{i}, k_{i}} d s= & \frac{\left(\prod_{i=1}^{N} \lambda_{k_{i}}^{-1} q_{k_{i}, k_{i}}^{\ell_{i}, \ell_{i}}\right)^{\frac{1}{2}}}{\sum_{i=1}^{N} d_{\ell_{i}} \lambda_{k_{i}}}\left\{\prod_{i=1}^{N} \lambda_{k_{i}}^{\frac{1}{2} \delta_{0}} \mathcal{O}\left(\varepsilon^{2-r-3 \delta_{0}}\right)+\sum_{i=1}^{N} \lambda_{k_{i}}^{\frac{1}{2}} \mathcal{O}\left(\varepsilon^{1-r}\right)\right\} \\
& +\frac{1}{\sum_{i=1}^{N} d_{\ell_{i}} \lambda_{k_{i}}} \sum_{\substack{j_{1}, j_{2}=1, j_{1} \neq j_{2}}}^{N} q_{k_{j_{1}}, k_{j_{2}}}^{\ell_{j_{1}, \ell_{j_{2}}}} \int_{0}^{t} X \prod_{\substack{i=1, i \notin\left\{j_{1}, j_{2}\right\}}}^{N} \mathcal{Z}_{\ell_{i}, k_{i}} d s .
\end{aligned}
$$

We use $\sum_{i=1}^{N} d_{\ell_{i}} \lambda_{k_{i}} \geq c \prod_{i=1}^{N} \lambda_{k_{i}}^{1 / N}$ with $c=\prod_{i=1}^{N} d_{\ell_{j_{i}}}^{1 / N}$ and the equivalence of norms in $\mathbb{R}^{N}$ which implies for $C_{1}, C_{2}>0$

$$
C_{1}\left(\sum_{i=1}^{N} \lambda_{k_{i}}\right)^{\frac{1}{2}} \leq \sum_{i=1}^{N} \lambda_{k_{i}}^{\frac{1}{2}} \leq C_{2}\left(\sum_{i=1}^{N} \lambda_{k_{i}}\right)^{\frac{1}{2}}
$$

Hence,

$$
\begin{aligned}
\int_{0}^{t} X \prod_{i=1}^{N} \mathcal{Z}_{\ell_{i}, k_{i}} d s= & \left(\frac{1}{\sum_{i=1}^{N} d_{\ell_{i}} \lambda_{k_{i}}} \prod_{i=1}^{N} \lambda_{k_{i}}^{-1} q_{k_{i}, k_{i}}^{\ell_{i}, \ell_{i}}\right)^{\frac{1}{2}} \mathcal{O}\left(\varepsilon^{1-r}\right) \\
& +\frac{1}{\sum_{i=1}^{N} d_{\ell_{i}} \lambda_{k_{i}}} \sum_{\substack{j_{1}, j_{2}=1 \\
j_{1} \neq j_{2}}}^{N} q_{k_{j_{1}}, k_{j_{2}}}^{\ell_{j_{1}}, \ell_{j_{2}}} \int_{0}^{t} X \prod_{\substack{i=1, i \notin\left\{j_{1}, j_{2}\right\}}}^{N} \mathcal{Z}_{\ell_{i}, k_{i}} d s .
\end{aligned}
$$


In the case $N=3$, for example, Equation (62) takes the form

$$
\begin{aligned}
\int_{0}^{t} X \prod_{i=1}^{3} \mathcal{Z}_{\ell_{i}, k_{i}} d s= & \left(\frac{1}{\sum_{i=1}^{3} d_{\ell_{i}} \lambda_{k_{i}}} \prod_{i=1}^{3} \lambda_{k_{i}}^{-1} q_{k_{i}, k_{i}}^{\ell_{i}, \ell_{i}}\right)^{\frac{1}{2}} \mathcal{O}\left(\varepsilon^{1-r}\right) \\
& +\sum_{\substack{j_{1}, j_{2}=1 \\
j_{1} \neq j_{2}}}^{3} \frac{q_{k_{j_{1}}, k_{j_{2}}}^{\ell_{j_{1}}, \ell_{j_{2}}}}{d_{\ell_{j_{1}}} \lambda_{k_{j_{1}}}+d_{\ell_{j_{2}}} \lambda_{k_{j_{2}}}} \frac{\left(q_{j, j}^{i, i}\right)^{\frac{1}{2}}}{\lambda_{j}} \mathcal{O}\left(\varepsilon^{1-r}\right),
\end{aligned}
$$

where we used Equation (60) and $d_{\ell_{j_{1}}} \lambda_{k_{j_{1}}}+d_{\ell_{j_{2}}} \lambda_{k_{j_{2}}} \leq \sum_{i=1}^{3} d_{\ell_{i}} \lambda_{k_{i}}$ for $j_{1}, j_{2} \in$ $\{1,2,3\}$. The general case for $N \in\{5,7, \cdots\}$ follows similarly.

We prove the second part only for $N=2$ and we can proceed by induction. Applying Itô formula to $X \cdot \prod_{i=1}^{2} \mathcal{Z}_{\ell_{i}, k_{i}}$ and integrating from 0 to $t$, we obtain

$$
\begin{aligned}
\int_{0}^{t} X \prod_{i=1}^{2} \mathcal{Z}_{\ell_{i}, k_{i}} d s & =\frac{1}{\sum_{i=1}^{2} d_{\ell_{i}} \lambda_{k_{i}}}\left\{-\varepsilon^{2} X(t) \prod_{i=1}^{2} \mathcal{Z}_{\ell_{i}, k_{i}}(t)+\varepsilon^{2} \int_{0}^{t} G \prod_{i=1}^{2} \mathcal{Z}_{\ell_{i}, k_{i}} d s\right. \\
& \left.+\varepsilon \sum_{j=1}^{2} \int_{0}^{t} X \prod_{\substack{i=1 \\
i \neq j}}^{2} \mathcal{Z}_{\ell_{i}, k_{i}} d \tilde{W}_{\ell_{j}, k_{j}}+\int_{0}^{t} X d \tilde{W}_{\ell_{j_{1}}, k_{j_{1}}} d \tilde{W}_{\ell_{j_{2}}, k_{j_{2}}}\right\}
\end{aligned}
$$

Using Corollary 27 and Lemma 28 to obtain

$$
\begin{aligned}
\int_{0}^{t} X \prod_{i=1}^{2} \mathcal{Z}_{\ell_{i}, k_{i}} d s= & \frac{1}{\sum_{i=1}^{2} d_{\ell_{i}} \lambda_{k_{i}}}\left(\prod_{i=1}^{2} \lambda_{k_{i}}^{-1} q_{k_{i}, k_{i}}^{\ell_{i}, \ell_{i}}\right)^{\frac{1}{2}}\left\{\mathcal{O}\left(\varepsilon^{1-r-2 \delta_{0}}\right) \prod_{i=1}^{2} \lambda_{k_{i}}^{\frac{1}{2} \delta_{0}}\right. \\
& \left.+\mathcal{O}\left(\varepsilon^{1-r}\right) \sum_{i=1}^{2} \lambda_{k_{i}}^{\frac{1}{2}}\right\}+\frac{q_{k_{1}, k_{2}}^{\ell_{1}, \ell_{2}}}{\sum_{i=1}^{2} d_{\ell_{i}} \lambda_{k_{i}}} \int_{0}^{t} X d s .
\end{aligned}
$$

Using (61) with $\left(\sum_{i=1}^{2} d_{\ell_{i}} \lambda_{k_{i}}\right)^{\frac{1}{2}} \geq c \prod_{i=1}^{2} \lambda_{k_{i}}^{\frac{1}{4}}$ we obtain for $\delta_{0}<\frac{1}{2}$

$$
\int_{0}^{t} X \prod_{i=1}^{2} \mathcal{Z}_{\ell_{i}, k_{i}} d s=\left(\frac{1}{\sum_{i=1}^{2} d_{\ell_{i}} \lambda_{k_{i}}} \prod_{i=1}^{2} \lambda_{k_{i}}^{-1} q_{k_{i}, k_{i}}^{\ell_{i}, \ell_{i}}\right)^{\frac{1}{2}} \mathcal{O}\left(\varepsilon^{1-r}\right)+\frac{q_{k_{1}, k_{2}}^{\ell_{1}, \ell_{2}}}{\sum_{i=1}^{2} d_{\ell_{i}} \lambda_{k_{i}}} \int_{0}^{t} X d s
$$

For $N \in\{4,6, .$.$\} we apply Itô formula to the term X \prod_{i=1}^{N} \mathcal{Z}_{\ell_{i}, k_{i}}$ to obtain

$$
\begin{aligned}
\int_{0}^{t} X \prod_{i=1}^{N} \mathcal{Z}_{\ell_{i}, k_{i}} d s= & \frac{1}{\sum_{i=1}^{N} d_{\ell_{i}} \lambda_{k_{i}}}\left\{-\varepsilon^{2} X(t) \prod_{i=1}^{N} \mathcal{Z}_{\ell_{i}, k_{i}}(t)+\varepsilon^{2} \int_{0}^{t} G \prod_{i=1}^{N} \mathcal{Z}_{\ell_{i}, k_{i}} d s\right. \\
+ & \varepsilon \sum_{j=1}^{N} \int_{0}^{t} X \prod_{\substack{i=1, i \neq j}}^{N} \mathcal{Z}_{\ell_{i}, k_{i}} d \tilde{W}_{\ell_{j}, k_{j}} \\
& \left.+\sum_{j_{1} \neq j_{2}=1}^{N} \int_{0}^{t} X \prod_{\substack{i=1, i \neq j_{1} \neq j_{2}}}^{N} \mathcal{Z}_{\ell_{i}, k_{i}} d \tilde{W}_{\ell_{j_{1}}, k_{j_{1}}} d \tilde{W}_{\ell_{j_{2}}, k_{j_{2}}}\right\} .
\end{aligned}
$$


Using Corollary 27 and Lemma 28 to obtain as in the odd case before

$$
\begin{aligned}
\int_{0}^{t} X \prod_{i=1}^{N} \mathcal{Z}_{\ell_{i}, k_{i}} d s= & \left(\frac{1}{\sum_{i=1}^{N} d_{\ell_{i}} \lambda_{k_{i}}} \prod_{i=1}^{N} \lambda_{k_{i}}^{-1} q_{k_{i}, k_{i}}^{\ell_{i}, \ell_{i}}\right)^{\frac{1}{2}} \mathcal{O}\left(\varepsilon^{1-r}\right) \\
& +\sum_{\substack{j_{1}, j_{2}=1 \\
j_{1} \neq j_{2}}}^{N} \frac{q_{k_{j_{1}}, k_{j_{2}}}^{\ell_{j_{1}}, \ell_{j_{2}}}}{\sum_{i=1}^{N} d_{\ell_{i}} \lambda_{k_{i}}} \cdot \int_{0}^{t} X \prod_{\substack{i=1, i \notin\left\{j_{1}, j_{2}\right\}}}^{N} \mathcal{Z}_{\ell_{i}, k_{i}} d s
\end{aligned}
$$

The first factor in the sum is summable over $j_{1}$ and $j_{2}$ by Condition (13). Now, we can proceed by induction and apply the assertion for $N-2$ to obtain (59).

Lemma 32 Under Assumption [2, 因and [6 let $X$ be as in Lemma 29]. Then, for $\ell \in \mathbb{N}_{0}^{n}$ with $m \geq|\ell| \geq 1$, we obtain:

1- If one of the $\ell_{i}$ is odd, then

$$
P_{c} \int_{0}^{t} X\left(\mathcal{Z}^{s}\right)^{\ell} d \tau=\mathcal{O}\left(\varepsilon^{1-r}\right) .
$$

2-If all $\ell_{i}$ are even, then there is a constant $C_{\ell}$ such that

$$
P_{c} \int_{0}^{t} X\left(\mathcal{Z}^{s}\right)^{\ell} d \tau=C_{\ell} \int_{0}^{t} X d \tau+\mathcal{O}\left(\varepsilon^{1-r}\right),
$$

where $C_{\ell}$ is given by

$$
C_{\ell}=\prod_{i=1}^{n}\left(\frac{1}{2^{\ell_{i} / 2} d_{i}^{\ell_{i} / 2}} \sum_{k_{1}, ., k_{\ell_{i}}=1}^{\infty} \sum_{j \in \operatorname{Per}\left(\ell_{i}\right)} \prod_{\eta=1}^{\ell_{i} / 2} \frac{\mathrm{q}_{k_{j_{2 \eta-1}}, k_{j_{2 \eta}}}^{i, i}}{\lambda_{k_{j_{2 \eta-1}}}+\lambda_{k_{j_{2 \eta}}}} P_{c} \prod_{\eta=1}^{\ell_{i}} g_{k_{\eta}}\right) .
$$

Proof. From the definition of $\mathcal{Z}^{s}$ (cf. (27), we obtain

$$
\left(\mathcal{Z}^{s}\right)^{\ell}=\prod_{i=1}^{n} \mathcal{Z}_{i}^{\ell_{i}}=\prod_{i=1}^{n}\left(\sum_{j_{1}, \cdots, j_{\ell_{i}}=1}^{\infty} \prod_{k=1}^{\ell_{i}} \mathcal{Z}_{i, j_{k}} g_{j_{k}}\right) .
$$

We focus in the proof on the case $n=1$ and $n=2$ as they are needed for our applications. The general case follows similarly but it is technically more involved. For $n=1$ we have $\ell=\ell_{1}$ and

$$
P_{c} \int_{0}^{t} X\left(\mathcal{Z}^{s}\right)^{\ell} d \tau=P_{c} \sum_{j_{1}, \cdots, j_{\ell}=1}^{\infty} \prod_{k=1}^{\ell} g_{j_{k}} \int_{0}^{t} X \prod_{k=1}^{\ell} \mathcal{Z}_{1, j_{k}} d \tau .
$$

Now we consider two cases. First if $|\ell|$ is odd, then Lemma 29 with $N=|\ell|$ yields

$$
\int_{0}^{t} X\left(\mathcal{Z}^{s}\right)^{\ell} d \tau=\sum_{k_{1}=1}^{\infty} \cdots \sum_{k_{\ell}=1}^{\infty} A_{k_{1}, \cdots, k_{\ell}}^{1, \cdots, 1} \prod_{j=1}^{\ell} g_{k_{j}} \cdot \mathcal{O}\left(\varepsilon^{1-r}\right) .
$$

And then as the $A$ 's are summable

$$
P_{c} \int_{0}^{t} X\left(\mathcal{Z}^{s}\right)^{\ell} d \tau=\mathcal{O}\left(\varepsilon^{1-r}\right) .
$$


Secondly, if $|\ell|$ is even, then Lemma 29 implies

$$
\begin{aligned}
\int_{0}^{t} X\left(\mathcal{Z}^{s}\right)^{\ell} d \tau= & \sum_{k_{1}, \cdots, k_{\ell}=1}^{\infty} \frac{1}{2^{\frac{\ell}{2}} d_{1}^{\frac{\ell}{2}}} \sum_{j \in \operatorname{Per}(\ell)} \prod_{\eta=1}^{|\ell| / 2} \frac{q_{k_{j_{2 \eta-1}}, k_{j_{2 \eta}}}^{1}}{\lambda_{k_{j_{2 \eta}-1}}+\lambda_{k_{j_{2 \eta}}}} \prod_{\eta=1}^{\ell} g_{k_{\eta}} \int_{0}^{t} X d s \\
& +\sum_{k_{1}=1}^{\infty} \cdots \sum_{k_{\ell}=1}^{\infty} A_{k_{1}, \cdots, k_{\ell}}^{1, \cdots, 1} \prod_{\eta=1}^{\ell} g_{k_{\eta}} \mathcal{O}\left(\varepsilon^{1-r}\right) .
\end{aligned}
$$

As the $A$ 's are summable

$$
\begin{aligned}
P_{c} \int_{0}^{t} X\left(\mathcal{Z}^{s}\right)^{\ell} d \tau= & \sum_{k_{1}, \cdots, k_{\ell}=1}^{\infty} \frac{1}{2^{\frac{\ell}{2}}} d_{1}^{\frac{\ell}{2}} \sum_{j \in \operatorname{Per}(\ell)} \prod_{\eta=1}^{|\ell| / 2} \frac{q_{k_{j_{2 \eta-1}}, k_{j_{2} \eta}}^{1,1}}{\lambda_{k_{j_{2 \eta-1}}}+\lambda_{k_{j_{2 \eta}}}} P_{c} \prod_{\eta=1}^{\ell} g_{k_{\eta}} \int_{0}^{t} X d s \\
& +\mathcal{O}\left(\varepsilon^{1-r}\right) .
\end{aligned}
$$

For $n=2$, we have $N=|\ell|=\ell_{1}+\ell_{2}$ and from Equation (66)

$$
\int_{0}^{t} X\left(\mathcal{Z}^{s}\right)^{\ell} d \tau=\sum_{j_{1}=1}^{\infty} \cdots \sum_{j_{|\ell|}=1}^{\infty} \int_{0}^{t} X \prod_{k=1}^{|\ell|} \mathcal{Z}_{i_{k}, j_{k}} d \tau \prod_{k=1}^{|\ell|} g_{j_{k}},
$$

with $i_{1}=\cdots=i_{\ell_{1}}=1$ and $i_{\ell_{1}+1}=\cdots=i_{|\ell|}=2$. Similarly to the first part, we consider two cases. First if $|\ell|$ is odd, then we apply Lemma 29 to obtain

$$
\int_{0}^{t} X\left(\mathcal{Z}^{s}\right)^{\ell} d \tau=\sum_{j_{1}=1}^{\infty} \cdots \sum_{j_{|\ell|}=1}^{\infty} A_{k_{j_{1}}, \cdots, k_{j_{|\ell|} \mid}}^{i_{1}, \cdots, i_{|\ell|}} \prod_{k=1}^{|\ell|} g_{j_{k}} \mathcal{O}\left(\varepsilon^{1-r}\right) .
$$

As the $A$ 's are summable

$$
P_{c} \int_{0}^{t} X\left(\mathcal{Z}^{s}\right)^{\ell} d \tau=\mathcal{O}\left(\varepsilon^{1-r}\right) .
$$

In the second case, when $|\ell|$ is even, we apply Lemma 29 and analogously to the first case we obtain

$$
\begin{aligned}
\int_{0}^{t} X\left(\mathcal{Z}^{s}\right)^{\ell} d \tau= & \sum_{j_{1}, \ldots, j_{|\ell|}=1}^{\infty} \frac{1}{2^{\frac{|\ell|}{2}}} \sum_{j \in \operatorname{Per}(|\ell|)} \prod_{\eta=1}^{|\ell| / 2} \frac{q_{j_{k_{2 \eta}-1}, j_{k_{2 \eta}}}^{i_{k_{2 \eta}}, i_{k_{2 \eta}}}}{d_{i_{k_{2 \eta-1}}} \lambda_{j_{k_{2 \eta-1}}}+d_{i_{k_{2 \eta}}} \lambda_{j_{k_{2 \eta}}}} \prod_{k=1}^{|\ell|} g_{j_{k}} \int_{0}^{t} X d s \\
& +\sum_{j_{1}=1}^{\infty} \cdots \sum_{j_{|\ell|}=1}^{\infty} A_{k_{j_{1}}, \cdots, k_{j_{|\ell|} \mid}}^{i_{1}, \cdots, i_{|\ell|}} \prod_{k=1}^{|\ell|} g_{j_{k}} \mathcal{O}\left(\varepsilon^{1-r}\right) .
\end{aligned}
$$

We obtain

$$
\begin{aligned}
P_{c} \int_{0}^{t} X\left(\mathcal{Z}^{s}\right)^{\ell} d \tau= & \sum_{j_{1}, \ldots, j_{|\ell|}=1}^{\infty} \frac{1}{2^{\frac{\ell \ell \mid}{2}}} \sum_{j \in \operatorname{Per}(|\ell|)} \prod_{\eta=1}^{|\ell| / 2} \frac{q_{j_{k_{2 \eta-1}}, j_{k_{2 \eta}}}^{i_{k_{2 n}}, i_{k_{2 \eta}}}}{d_{i_{k_{2 \eta-1}}} \lambda_{j_{k_{2 \eta-1}}}+d_{i_{k_{2 \eta}}} \lambda_{j_{k_{2 \eta}}}} \\
& \times P_{c}\left(\prod_{k=1}^{|\ell|} g_{j_{k}}\right) \int_{0}^{t} X d s+\mathcal{O}\left(\varepsilon^{1-r}\right) .
\end{aligned}
$$

We can distinguish between two cases when $|\ell|$ is even. First one of $\ell_{1}$ and $\ell_{2}$ is odd. Here $q_{j_{\ell_{1}}, j \ell_{1}+1}^{i_{\ell_{1}}, i_{\ell_{1}}}=0$, where $i_{\ell_{1}}=1$ and $i_{\ell_{1}+1}=2$. Thus

$$
P_{c} \int_{0}^{t} X\left(\mathcal{Z}^{s}\right)^{\ell} d \tau=\mathcal{O}\left(\varepsilon^{1-r}\right) .
$$


In the second case when $\ell_{1}$ and $\ell_{2}$ are both even, we have

$$
\begin{aligned}
P_{c} \int_{0}^{t} X\left(\mathcal{Z}^{s}\right)^{\ell} d \tau \\
=P_{c} \times \sum_{j_{1}=1}^{\infty} \ldots \sum_{j_{\ell_{1}}=1}^{\infty} \frac{1}{\left(2 d_{1}\right)^{\frac{\ell_{1}}{2}}} \sum_{j \in \operatorname{Per}\left(\ell_{1}\right)} \prod_{\eta=1}^{\ell_{1} / 2} \frac{q_{j_{k_{2 \eta}-1}, j_{k_{2 \eta} \eta}}^{1,1}}{\lambda_{j_{k_{2 \eta-1}}}+\lambda_{j_{k_{2} \eta}}} \prod_{k=1}^{\ell_{1}} g_{j_{k}} \\
\quad \times \sum_{j_{1}=1}^{\infty} \ldots \sum_{j_{\ell_{2}}=1}^{\infty} \frac{1}{\left(2 d_{2}\right)^{\frac{\ell_{2}}{2}}} \sum_{j \in \operatorname{Per}\left(\ell_{2}\right)} \prod_{\eta=1}^{\ell_{2} / 2} \frac{q_{j_{k_{2 \eta-1}}, j_{k_{2 \eta}}}^{2,2}}{\lambda_{j_{k_{2 \eta-1}}}+\lambda_{j_{k_{2 \eta}}}} \prod_{k=1}^{\ell_{2}} g_{j_{k}} \int_{0}^{t} X d s \\
\quad+\mathcal{O}\left(\varepsilon^{1-r}\right) \\
=\prod_{i=1}^{2} \sum_{j_{1}=1}^{\infty} \ldots \sum_{j_{\ell_{i}}=1}^{\infty} \frac{1}{\left(2 d_{i}\right)^{\frac{\ell_{i}}{2}}} \sum_{j \in \operatorname{Per}\left(\ell_{1}\right)} \prod_{\eta=1}^{\ell_{i} / 2} \frac{q_{j_{k_{2 \eta-1}}, j_{k_{2 \eta}}}^{2,2}}{\lambda_{j_{k_{2 \eta-1}}}+\lambda_{j_{k_{2 \eta}}}} \int_{0}^{t} X d s \cdot P_{c} \prod_{k=1}^{|\ell|} g_{j_{k}} \\
\quad+\mathcal{O}\left(\varepsilon^{1-r}\right) .
\end{aligned}
$$

The general case for $n>2$ follows in a similar way, as the random variables $\left(\sum_{j_{1}, \cdots, j_{\ell_{i}}=1}^{\infty} \prod_{k=1}^{\ell_{i}} \mathcal{Z}_{i, j_{k}} g_{j_{k}}\right)_{i=1,2, \ldots, n}$ are independent, and we can thus glue together the individual averaging results as above.

\section{Proof of the Approximation Theorem I}

Lemma 33 Let Assumptions因回 and廌 hold. Then

$$
a_{i}(t)=a_{i}(0)+\int_{0}^{t} \mathcal{F}_{i}(a) d \tau+\sum_{|\ell|=2,4, . .} \frac{C_{\ell}}{\ell !} \int_{0}^{t} D^{\ell} \mathcal{F}_{i}(a) d \tau+\tilde{R}(t),
$$

where $C_{\ell}$ was defined in (65) and the error is bounded by $\tilde{R}=\mathcal{O}\left(\varepsilon^{1-2 m \kappa-\kappa_{0}}\right)$.

Proof. The mild formulation of (37) and Lemma 22 with $\rho=0$ yields

$$
\psi(t)=\mathcal{Z}^{s}(t)+e^{\varepsilon^{-2} t \mathcal{A}} \psi(0)+\mathcal{O}\left(\varepsilon^{2-m \kappa}\right)=: \mathcal{Z}^{s}(t)+y(t)+R(t),
$$

where

$$
y(t)=e^{\varepsilon^{-2} t \mathcal{A}} \psi(0) \text { and } R(t)=\mathcal{O}\left(\varepsilon^{2-m \kappa}\right) .
$$

Substituting from (68) into (36), yields

$$
a_{i}(t)=a_{i}(0)+\int_{0}^{t} \mathcal{F}_{i}\left(a+\mathcal{Z}^{s}+y+R\right)(\tau) d \tau .
$$

Taylor's expansion for the polynomial $\mathcal{F}_{i}: \mathcal{L}_{n}^{p} \rightarrow \mathbb{R}$ yields

$$
a_{i}(t)=a_{i}(0)+\int_{0}^{t} \mathcal{F}_{i}\left(a+\mathcal{Z}^{s}\right)(\tau) d \tau+R_{i}^{(1)}(t),
$$

where $R^{(1)}(t)$ is given by

$$
R_{i}^{(1)}(t)=\sum_{|\ell| \geq 1} P_{c} \int_{0}^{t} \frac{D^{\ell} \mathcal{F}_{i}\left(a+\mathcal{Z}^{s}\right)}{\ell !}(y+R)^{\ell} d \tau .
$$


We see later that $R^{(1)}$ is small, as all terms contain at least one $R=\mathcal{O}\left(\varepsilon^{2-m \kappa}\right)$.

Taylor's expansion again for the polynomial $\mathcal{F}_{i}: \mathcal{L}_{n}^{p} \rightarrow \mathbb{R}$, yields

$$
a_{i}(t)=a_{i}(0)+\sum_{|\ell| \geq 0} P_{c} \int_{0}^{t} \frac{D^{\ell} \mathcal{F}_{i}(a)}{\ell !}\left(\mathcal{Z}^{s}\right)^{\ell} d \tau+R_{1}(t) .
$$

Applying the Averaging-Lemma 32 yields

$$
a_{i}(t)=a_{i}(0)+\sum_{|\ell| \geq 0} \frac{C_{\ell}}{\ell !} \int_{0}^{t} D^{\ell} \mathcal{F}_{i}(a) d \tau+\mathcal{O}\left(\varepsilon^{1-m_{i} \kappa}\right)+R_{i}^{(1)}(t),
$$

where $C_{0}=1$ and $C_{\ell}=0$ if one $\ell_{i}$ is odd. Thus

$$
a_{i}(t)=a_{i}(0)+\sum_{|\ell|=0,2,4, . .} \frac{C_{\ell}}{\ell !} \int_{0}^{t} D^{\ell} \mathcal{F}_{i}(a) d \tau+\tilde{R}_{i}(t),
$$

where $\tilde{R}(t)=R^{(1)}(t)+\mathcal{O}\left(\varepsilon^{1-m \kappa}\right)$.

To bound $\tilde{R}$ we use Lemmas 25 and 23 and Assumption 5 .

Definition 34 Define the set $\Omega^{*} \subset \Omega$ such that all the following estimates hold on $\Omega^{*}$

$$
\begin{gathered}
\sup _{\left[0, \tau^{*}\right]}\|\psi-\mathcal{Q}\|_{\mathcal{L}_{n}^{p}}<C \varepsilon^{2-m \kappa-\kappa}, \\
\sup _{\left[0, \tau^{*}\right]}\|\psi\|_{\mathcal{L}_{n}^{p}}<C \varepsilon^{-\frac{3}{2} \kappa_{0}}, \\
\sup _{\left[0, \tau^{*}\right]}\|\tilde{R}\|_{\mathcal{L}_{n}^{p}}<C \varepsilon^{1-2 m \kappa-\kappa},
\end{gathered}
$$

and

$$
\sup _{\left[0, T_{1}\right]}|b| \leq \tilde{C}_{0}
$$

Proposition $35 \Omega^{*}$ has approximately probability 1.

\section{Proof.}

$$
\begin{gathered}
\mathbb{P}\left(\Omega^{*}\right) \geq 1-\mathbb{P}\left(\sup _{\left[0, \tau^{*}\right]}\|\psi-\mathcal{Q}\|_{\mathcal{L}_{n}^{p}} \geq C \varepsilon^{2-m \kappa-\kappa}\right)-\mathbb{P}\left(\sup _{\left[0, \tau^{*}\right]}\|\psi\|_{\mathcal{L}_{n}^{p}} \geq C \varepsilon^{-\frac{3}{2} \kappa_{0}}\right) \\
-\mathbb{P}\left(\sup _{\left[0, \tau^{*}\right]}\|\tilde{R}\|_{\mathcal{L}_{n}^{p}} \geq C \varepsilon^{1-2 m \kappa-\kappa}\right)-\mathbb{P}\left(\sup _{\left[0, T_{1}\right]}|b|>\tilde{C}_{0}\right)
\end{gathered}
$$

Using Chebychev inequality and Lemmas 22, 33 and Corollary 24, we obtain for $\kappa>\kappa_{0}$ and sufficiently large $q>\frac{2 p}{\left(\kappa-\kappa_{0}\right)}>0$

$$
\begin{aligned}
\mathbb{P}\left(\Omega^{*}\right) & \geq 1-C\left[\varepsilon^{q \kappa}+\varepsilon^{\frac{1}{2} q \kappa}+\varepsilon^{q\left(\kappa-\kappa_{0}\right)}\right]-\mathbb{P}\left(\sup _{\left[0, T_{1}\right]}|b|>\tilde{C}_{0}\right) \\
& \geq 1-C \varepsilon^{\frac{1}{2} q\left(\kappa-\kappa_{0}\right)}-\mathbb{P}\left(\sup _{\left[0, T_{1}\right]}|b|>\tilde{C}_{0}\right) \\
& \geq 1-C \varepsilon^{p},
\end{aligned}
$$

where $\tilde{C}_{0}$ is chosen sufficiently large $\left(\sup _{\left[0, T_{1}\right]}|b| \leq C\right.$ by Assumption 8$)$. 
Theorem 36 Assume that Assumptions 5 and 8 hold. Suppose $a(0)=\mathcal{O}(1)$ and $\psi(0)=\mathcal{O}(1)$. Let $b$ be a solution of (3) and a as defined in (67). If the initial conditions satisfy $a(0)=b(0)$, then for $\kappa<\frac{1}{2 m+1}$ we obtain

$$
\sup _{t \in\left[0, T_{1} \wedge \tau^{*}\right]}|a(t)-b(t)| \leq C \varepsilon^{1-2 m \kappa-\kappa} \quad \text { on } \Omega^{*},
$$

and

$$
\sup _{t \in\left[0, T_{1} \wedge \tau^{*}\right]}|a(t)| \leq C \quad \text { on } \Omega^{*} .
$$

We note that all norms in a finite dimensional space are equivalent. Thus for simplicity of notation we always use the standard Euclidean norm.

Proof. Subtracting (3) from (67) and defining

$$
h:=a-b,
$$

we obtain

$$
h(t)=\sum_{|\ell|=0,2,4, . .} \frac{C_{\ell}}{\ell !} \int_{0}^{t}\left[D^{\ell} \mathcal{F}_{i}(h+b)-D^{\ell} \mathcal{F}_{i}(b)\right] d \tau+\tilde{R}(t),
$$

where the error $\tilde{R}$ is bounded by $\tilde{R}=\mathcal{O}\left(\varepsilon^{1-2 m \kappa}\right)$.

Define $Q$ as

$$
Q:=h-\tilde{R} .
$$

From Equation (79) we obtain

$$
\partial_{t} Q=\sum_{|\ell|=0,2,4, . .} \frac{C_{\ell}}{\ell !} D^{\ell}\left[\mathcal{F}_{i}(Q+\tilde{R}+b)-D^{\ell} \mathcal{F}_{i}(b)\right] .
$$

Taking the scalar product $\langle Q, \cdot\rangle$ on both sides, yields

$$
\frac{1}{2} \partial_{t}|Q|^{2}=\sum_{|\ell|=0,2,4, . .} \frac{C_{\ell}}{\ell !}\left\langle D^{\ell} \mathcal{F}_{i}(Q+\tilde{R}+b)-D^{\ell} \mathcal{F}_{i}(b), Q\right\rangle .
$$

Using Young and Cauchy-Schwarz inequalities, where $\mathcal{F}$ is a polynomial of degree $m$, we obtain

$$
\frac{1}{2} \partial_{t}|Q|^{2} \leq C\left(1+|Q|^{m-1}+|\tilde{R}|^{m-1}+|b|^{m-1}\right)\left(|Q|^{2}+|\tilde{R}|^{2}\right) .
$$

As long as $|Q|<1$, using Equations (73) and (74), we obtain for $\kappa<\frac{1}{2 m+1}$

$$
\frac{1}{2} \partial_{t}|Q|^{2} \leq c|Q|^{2}+C \varepsilon^{2-2(2 m+1) \kappa} \text { on } \Omega^{*},
$$

Using Gronwall's lemma, we obtain for $t \leq \tau^{*} \wedge T_{1} \leq T_{0}$

$$
|Q(t)|^{2} \leq C \varepsilon^{1-(2 m+1) \kappa} e^{2 c T_{0}},
$$

and thus $|Q(t)|<1$ for $t \leq \tau^{*} \wedge T_{1}$. Taking supremum on $\left[0, \tau^{*} \wedge T_{1}\right]$

$$
\sup _{t \in\left[0, \tau^{*} \wedge T_{1}\right]}|Q(t)|^{2} \leq C \varepsilon^{1-(2 m+1) \kappa} \text { on } \Omega^{*} \text {. }
$$


Hence,

$$
\begin{aligned}
\sup _{\left[0, \tau^{*} \wedge T_{1}\right]}|a-b| & =\sup _{\left[0, \tau^{*} \wedge T_{1}\right]}|Q-\tilde{R}| \leq \sup _{\left[0, \tau^{*} \wedge T_{1}\right]}|Q(t)|+\sup _{\left[0, \tau^{*} \wedge T_{1}\right]}|\tilde{R}| \\
& \leq C \varepsilon^{1-(2 m+1) \kappa} \text { on } \Omega^{*} .
\end{aligned}
$$

We finish the proof by using (78), (80) and

$$
\sup _{\left[0, \tau^{*} \wedge T_{1}\right]}|a| \leq \sup _{\left[0, \tau^{*} \wedge T_{1}\right]}|a-b|+\sup _{\left[0, \tau^{*} \wedge T_{1}\right]}|b| \leq C .
$$

Now we can collect the results obtained previously to prove the main result of Theorem 17 and Corollary 18 for the system of SPDE (10).

Proof of Theorem 17. Using (30) and triangle inequality, we obtain

$$
\begin{aligned}
\sup _{t \in\left[0, T_{1} \wedge \tau^{*}\right]}\|u(t)-b(t)-\mathcal{Q}(t)\|_{\mathcal{L}_{n}^{p}} & \leq \sup _{\left[0, T_{1} \wedge \tau^{*}\right]}\|a-b\|_{\mathcal{L}_{n}^{p}}+\sup _{\left[0, T_{1} \wedge \tau^{*}\right]}\|\psi-\mathcal{Q}\|_{\mathcal{L}_{n}^{p}} \\
& \leq C \sup _{\left[0, T_{1} \wedge \tau^{*}\right]}|a-b|+\sup _{\left[0, \tau^{*}\right]}\|\psi-\mathcal{Q}\|_{\mathcal{L}_{n}^{p} .} .
\end{aligned}
$$

From (71) and (76), we obtain

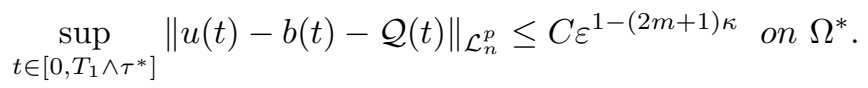

Hence,

$$
\mathbb{P}\left(\sup _{t \in\left[0, T_{1} \wedge \tau^{*}\right]}\|u(t)-b(t)-\mathcal{Q}(t)\|_{\mathcal{L}_{n}^{p}}>C \varepsilon^{1-(2 m+1) \kappa}\right) \leq 1-\mathbb{P}\left(\Omega^{*}\right) .
$$

Using (75), yields (33).

Proof of Corollary 18. We note that by the semigroup estimate based on Assumptions 3 and Equation (8)

$$
\begin{aligned}
\|\psi(t)\|_{\mathcal{L}_{n}^{m p}} & \leq\left\|e^{\varepsilon^{-2} t \mathcal{A}} \psi(0)\right\|_{\mathcal{L}_{n}^{m p}}+\left\|\mathcal{Z}^{s}(t)\right\|_{\mathcal{L}_{n}^{m p}}+\frac{1}{\varepsilon^{\rho}}\left\|\int_{0}^{t} e^{\varepsilon^{-2} \mathcal{A}_{s}(T-\tau)} \mathcal{F}^{s}(u) d \tau\right\|_{\mathcal{L}_{n}^{m p}} \\
& \leq e^{-\varepsilon^{-2} t \omega}\|\psi(0)\|_{\mathcal{L}_{n}^{m p}}+\left\|\mathcal{Z}^{s}(t)\right\|_{\mathcal{L}_{n}^{m p}}+C \varepsilon^{2-\rho} \sup _{\tau \in\left[0, \tau^{*}\right]}\left(1+\|u\|_{\mathcal{L}_{n}^{p m}}^{m}\right)
\end{aligned}
$$

where we used Assumption 5. Thus by the definition of $\tau^{*}$ and the bounds on $\mathcal{Z}^{s}$ (cf. (43)) we obtain on $\Omega^{*}$

$$
\sup _{t \in\left[0, \tau^{*}\right]}\|\psi(t)\|_{\mathcal{L}_{n}^{m p}} \leq C \varepsilon^{-\kappa_{0}} .
$$

Thus from the Theorem 36 we derive

$$
\Omega \supset\left\{\tau^{*}>T_{1}\right\} \supseteq\left\{\sup _{\left[0, T_{1} \wedge \tau^{*}\right]}\|u\|_{\mathcal{L}_{n}^{m p}}<\varepsilon^{-\kappa}\right\} \supseteq \Omega^{*} .
$$

Hence,

$$
\begin{aligned}
\sup _{t \in\left[0, T_{1}\right]}\|u(t)-b(t)-\mathcal{Q}(t)\|_{\mathcal{L}_{n}^{m p}} & \leq \sup _{\left[0, T_{1}\right]}\|a-b\|_{\mathcal{L}_{n}^{m p}}+\sup _{\left[0, T_{1}\right]}\|\psi-\mathcal{Q}\|_{\mathcal{L}_{n}^{m p}} \\
& \leq C \sup _{\left[0, T_{1}\right]}|a-b|+\sup _{\left[0, \tau^{*}\right]}\|\psi-\mathcal{Q}\|_{\mathcal{L}_{n}^{m p}} .
\end{aligned}
$$

Proceeding as in the proof of Theorem 17 we bound the error in $\mathcal{L}_{n}^{m p}$. 


\subsection{Application of Approximation Theorem I}

In this subsection we consider all examples with non-homogeneous Neumann boundary condition on $[0,1]^{2}$. Here the eigenfunctions are

$$
g_{k_{1}, k_{2}}=\left\{\begin{array}{cll}
1 & \text { if } & k_{1}=k_{2}=0 \\
2 \cos \left(\pi k_{1} x\right) \cos \left(\pi k_{2} y\right) & \text { if } & k_{1}, k_{2}>0
\end{array}\right.
$$

The eigenvalues of the operator $-\mathcal{A}_{i}=-d_{i}\left(\partial_{x}^{2}+\partial_{y}^{2}\right)$ are $\lambda_{k_{1}, k_{2}}=\pi^{2}\left(k_{1}^{2}+k_{2}^{2}\right)$. Define $f_{\ell}(z)$ as

$$
f_{\ell}(z)=\left\{\begin{array}{ccc}
1 & \text { if } \quad \ell=0 \\
\sqrt{2} \cos (\pi \ell z) & \text { if } \quad \ell>0
\end{array}\right.
$$

Now $g_{k}(x, y)=f_{k_{1}}(x) f_{k_{2}}(y)$ for $k \in \mathbb{N}_{0}^{2}$.

\subsubsection{Physical Application (Nonlinear Heat Eq.)}

The heat equation plays a significant role in several areas of science including mathematics, probability theory and financial mathematics. In probability theory for instance, the heat equation is used for studying Brownian motion via the Fokker-Planck equation.

To apply our main Theorem 17, we consider the following nonlinear heat Equation with stochastic Neumann boundary condition.

$$
\begin{aligned}
\partial_{t} u & =\varepsilon^{-2}\left(\partial_{x}^{2}+\partial_{y}^{2}\right) u+u-u^{3} \text { for } 0 \leq x \leq 1,0 \leq y \leq 1 \\
\partial_{x} u(t, x, 0) & =\sigma_{\varepsilon} \partial_{t} W_{1}(t, x), \partial_{x} u(t, x, 1)=\sigma_{\varepsilon} \partial_{t} W_{2}(t, x) \text { for } x \in(0,1) \\
\partial_{y} u(t, 0, y) & =\sigma_{\varepsilon} \partial_{t} W_{3}(t, x), \partial_{y} u(t, 1, y)=\sigma_{\varepsilon} \partial_{t} W_{4}(t, y) \text { for } y \in(0,1)
\end{aligned}
$$

Define $W_{i}(t)$ for $i=1,2,3,4$ as $W_{i}(t)=\sum_{j=1}^{\infty} \alpha_{i, j} \beta_{i, j}(t) f_{j}$ and $\mathcal{N}=\{1\}$.

Our main Theorem[17 states that the solution of the nonlinear heat equation (83) with $\sigma_{\varepsilon}=\varepsilon^{-1}$ is well approximated by

$$
u(t, x, y)=b(t)+\mathcal{Z}^{s}(t, x, y)+\mathcal{O}\left(\varepsilon^{1-}\right),
$$

where $b$ is the solution of

$$
\partial_{t} b=\left(1-3 C_{2}\right) b-b^{3}
$$

and $C_{2}$ is a constant given by $C_{2}=\sum_{k, j=1}^{\infty} \frac{q_{k, j}}{\lambda_{k}+\lambda_{j}} P_{c}\left(g_{k} g_{j}\right)$.

We calculate

$$
P_{c}\left(g_{k} g_{j}\right)= \begin{cases}\frac{1}{2} & \text { if } k_{1}=j_{1}, k_{2}=j_{2} \\ 0 & \text { otherwise }\end{cases}
$$

and

$$
\begin{aligned}
q_{k, j}= & \delta_{k_{1}, j_{1}} \alpha_{1, k_{1}}^{2} f_{k_{2}}(0) f_{j_{2}}(0)+\delta_{k_{1}, j_{1}} \alpha_{2, k_{1}}^{2} f_{k_{2}}(1) f_{j_{2}}(1) \\
& +\delta_{k_{2}, j_{2}} \alpha_{3, k_{2}}^{2} f_{k_{1}}(0) f_{j_{1}}(0)+\delta_{k_{2}, j_{2}} \alpha_{4, k_{1}}^{2} f_{k_{1}}(1) f_{j_{1}}(1) .
\end{aligned}
$$

Thus

$$
C_{2}=\frac{1}{2 \pi^{2}} \sum_{k_{1}, k_{2}=1}^{\infty} \frac{1}{k_{1}^{2}+k_{2}^{2}}\left(\alpha_{1, k_{1}}^{2}+2 \alpha_{2, k_{1}}^{2}+\alpha_{3, k_{2}}^{2}+2 \alpha_{4, k_{2}}^{2}\right) .
$$


If we choose for any $\mu>0$ that $\alpha_{i, k}^{2} \leq C|k|^{-2 \mu}$ for $i=1, \ldots, 4$ and all $k \in \mathbb{N}$, then $C_{2}$ is finite and furthermore, all summability conditions are satisfied.

Let us finally check the bound on $b$. Taking the product with $b$ on both sides of (84), yields

$$
\frac{1}{2} \partial_{t}|b|^{2}=C|b|^{2}-|b|^{4} \leq C|b|^{2} .
$$

Using Gronwall's lemma, we obtain for $0 \leq t \leq T_{0}$ that

$$
\sup _{\left[0, T_{0}\right]}|b|^{2} \leq|b(0)|^{2} e^{C T_{0}} .
$$

Thus Assumption 8 is always true for deterministic initial conditions if we choose $C_{0}$ sufficiently large.

\subsubsection{Chemical Application}

A simple archetypical example for a reaction-diffusion system is a cubic autocatalytic reaction between two chemicals according to the rule $A+B \rightarrow 2 B$ with rate $r=\rho u_{1} u_{2}^{2}$.

Denoting by $u_{1}$ and $u_{2}$ the concentration of $A$ and $B$, respectively. The two species satisfy the equations:

$$
\partial_{t} u_{1}=\frac{1}{\varepsilon^{2}} \Delta u_{1}-\rho u_{1} u_{2}^{2} \quad \& \quad \partial_{t} u_{2}=\frac{d}{\varepsilon^{2}} \Delta u_{2}+\rho u_{1} u_{2}^{2} .
$$

with respect to stochastic boundary conditions for $i=1,2$

$\partial_{x} u_{i}(t, x, 0)=\sigma_{\varepsilon} \partial_{t} W_{i_{1}}(t, x), \partial_{x} u_{i}(t, x, 1)=\sigma_{\varepsilon} \partial_{t} W_{i_{2}}(t, x)$ for $x \in(0,1)$

$\partial_{y} u_{i}(t, 0, y)=\sigma_{\varepsilon} \partial_{t} W_{i_{3}}(t, x), \partial_{y} u_{i}(t, 1, y)=\sigma_{\varepsilon} \partial_{t} W_{i_{4}}(t, y)$ for $y \in(0,1),(86)$

where $W_{i_{j}}(t)=\sum_{k=1}^{\infty} \alpha_{i_{j}, k} \beta_{i_{j}, k}(t) f_{k}$ for $j=1, \ldots, 4$, and $f_{k}$ defined as before.

We define $\mathcal{N}=\left\{\left(\begin{array}{l}1 \\ 0\end{array}\right),\left(\begin{array}{l}0 \\ 1\end{array}\right)\right\}$ and take $\sigma_{\varepsilon}=\varepsilon$.

Then our main theorem states that

$$
\begin{gathered}
u(t)=b(t)+\mathcal{Z}^{s}(t)+\mathcal{O}\left(\varepsilon^{1-}\right), \\
\text { with } u=\left(\begin{array}{c}
u_{1} \\
u_{2}
\end{array}\right), \quad b=\left(\begin{array}{c}
b_{1} \\
b_{2}
\end{array}\right) \text {, and } \mathcal{Z}^{s}=\left(\begin{array}{c}
\mathcal{Z}_{1}^{s} \\
\mathcal{Z}_{2}^{s}
\end{array}\right),
\end{gathered}
$$

where $b_{1}$ and $b_{2}$ are the solutions of

$$
\begin{aligned}
& \partial_{t} b_{1}=-\rho b_{1} b_{2}^{2}-\rho C_{2} b_{1} \\
& \partial_{t} b_{2}=\rho b_{1} b_{2}^{2}+\rho C_{2} b_{1},
\end{aligned}
$$

with

$$
C_{2}=\frac{1}{2 \pi^{2}} \sum_{k_{1}, k_{2}=1}^{\infty} \frac{1}{k_{1}^{2}+k_{2}^{2}}\left(\alpha_{2_{1}, k_{1}}^{2}+2 \alpha_{2_{2}, k_{2}}^{2}+2 \alpha_{2_{3}, k_{1}}^{2}+\alpha_{2_{4}, k_{2}}^{2}\right) .
$$

We note that high fluctuations in combination with fast diffusion lead to effective new terms describing the transformation of $b_{1}$ to $b_{2}$. Although both terms 
individually do not change the average $\int u_{i} d x=b_{i}$, their nonlinear combination does.

Let us check the bound on $b$ from Assumption 8 , We note that

$$
\sum_{i=1}^{2} \partial_{t} b_{i}=0 \quad \text { and thus } \quad \sum_{i=1}^{2} b_{i}(t)=\sum_{i=1}^{2} b_{i}(0)=C_{0} .
$$

As $b_{1}(t) \geq 0$ and thus $b_{2}(t) \geq b_{2}(0) \geq 0$, we have $0 \leq b_{i}(t) \leq \sum_{i=1}^{2} b_{i}(t) \leq C_{0}$.

Hence, for all times $t>0$ we obtain $\|b(t)\|=\left(\sum_{i=1}^{2} b_{i}^{2}(t)\right)^{1 / 2} \leq C_{0} \sqrt{2}$.

\section{Proof of the Approximation Theorem II}

In this section, we use many ideas and lemmas of the previous sections, as the main ideas are similar.

Lemma 37 Let Assumption 5 holds. Then for $R^{(2)}$ defined in (39) as

$$
R_{i}^{(2)}=\sum_{|\ell| \geq 1} \int_{0}^{t} \frac{D^{\ell} \mathcal{F}_{i}(a)}{\ell !} P_{c}(\varepsilon \psi)^{\ell} d \tau
$$

we have $R^{(2)}=\mathcal{O}\left(\varepsilon^{1-m \kappa}\right)$.

Proof. Using Assumption 5

$$
\begin{aligned}
\mathbb{E} \sup _{\left[0, \tau^{*}\right]}\left|R_{i}^{(2)}\right|^{p} & \leq C \sum_{|\ell| \geq 1} \frac{1}{\ell !} \mathbb{E} \sup _{\left[0, \tau^{*}\right]} \int_{0}^{t}\left|D^{\ell} \mathcal{F}_{i}(a)\right|\|\varepsilon \psi\|_{\mathcal{L}_{n}^{\ell \mid}}^{|\ell| p} d \tau \\
& \leq C \sum_{|\ell| \geq 1} \frac{1}{\ell !}\left[1+\varepsilon^{(\ell-m) p \kappa}\right] \varepsilon^{\ell p(1-\kappa)} \\
& \leq C \varepsilon^{1-m \kappa} .
\end{aligned}
$$

Definition 38 Define the set $\stackrel{* *}{\Omega} \subset \Omega$ such that for sufficiently large $\zeta \gg 1$ all the following estimates hold on $\stackrel{* *}{\Omega}$

$$
\begin{gathered}
\sup _{\left[0, \tau^{*}\right]}\|\psi-\mathcal{Q}\|_{\mathcal{L}_{n}^{p}}<C \varepsilon^{1-m \kappa-\kappa}, \\
\sup _{\left[0, \tau^{*}\right]}\|\psi\|_{\mathcal{L}_{n}^{p}}<C \varepsilon^{-\frac{3}{2} \kappa_{0}} \\
\sup _{\left[0, \tau^{*}\right]}\left|R^{(2)}\right|<C \varepsilon^{1-m \kappa-\kappa}
\end{gathered}
$$

and

$$
\sup _{\left[0, T_{1}\right]}|b|^{m-1} \leq \ln \left(\varepsilon^{-\frac{1}{\zeta}}\right)
$$


Proposition $39 \stackrel{* *}{\Omega}$ has approximately probability 1.

Proof.

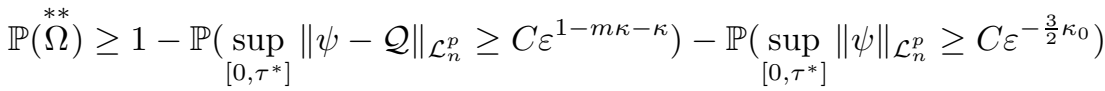

$$
\begin{aligned}
& -\mathbb{P}\left(\sup _{\left[0, \tau^{*}\right]}\left|R^{(2)}\right| \geq C \varepsilon^{1-m \kappa-\kappa}\right)-\mathbb{P}\left(\sup _{\left[0, T_{1}\right]}|b|^{m-1}>\ln \left(\varepsilon^{-\frac{1}{\zeta}}\right)\right) .
\end{aligned}
$$

Using Chebychev inequality and Lemmas 22, 37 and Corollary 24, we obtain for $\kappa>\kappa_{0}$ and sufficiently large $q>\frac{2 p}{\left(\kappa-\kappa_{0}\right)}>0$

$$
\begin{aligned}
\mathbb{P}(\stackrel{* *}{\Omega}) & \geq 1-C\left[\varepsilon^{q \kappa}+\varepsilon^{\frac{1}{2} q \kappa}+\varepsilon^{q\left(\kappa-\kappa_{0}\right)}\right]-\mathbb{P}\left(\sup _{\left[0, T_{1}\right]}|b|^{m-1}>\ln \left(\varepsilon^{-\frac{1}{\zeta}}\right)\right) \\
& \geq 1-C \varepsilon^{\frac{1}{2} q\left(\kappa-\kappa_{0}\right)}-\mathbb{P}\left(\sup _{\left[0, T_{1}\right]}|b|^{m-1}>\ln \left(\varepsilon^{-\frac{1}{\zeta}}\right)\right) \\
& \geq 1-C \varepsilon^{\delta \kappa}
\end{aligned}
$$

where we used Assumption 9 ,

Theorem 40 Assume that Assumptions 4 囵 and 9 hold. Suppose $a(0)=\mathcal{O}(1)$ and $\psi(0)=\mathcal{O}(1)$. Let $b \in \mathcal{N}$ be a solution of (5) and $a \in \mathcal{N}$ as defined in (38). If the initial conditions satisfy $a(0)=b(0)$, then for $\kappa<\frac{1}{m+2}$ we obtain

$$
\sup _{t \in\left[0, \tau^{*} \wedge T_{1}\right]}|a(t)-b(t)| \leq C \varepsilon^{1-(m+2) \kappa} \quad \text { on } \stackrel{* *}{\Omega} .
$$

Proof. We follow the same steps as in the proof of Lemma 36 until Equation (81) to obtain

$$
\frac{1}{2} \partial_{t}|Q|^{2} \leq C\left(1+|Q|^{m-1}+\left|R^{(2)}\right|^{m-1}+|b|^{m-1}\right)\left(|Q|^{2}+\left|R^{(2)}\right|^{2}\right) .
$$

As long as $|Q|<1$, using Equations (89) and (90), we obtain

$$
\frac{1}{2} \partial_{t}|Q(t)|^{2} \leq c\left(1+\ln \left(\varepsilon^{-\frac{1}{\zeta}}\right)\right)|Q(t)|^{2}+C \varepsilon^{2-2(m+1) \kappa} \text { on } \stackrel{* *}{\Omega} .
$$

Using Gronwall's lemma, we obtain for $t \leq \tau^{*} \wedge T_{1} \leq T_{0}$

$$
\begin{aligned}
|Q(t)|^{2} & \leq C \varepsilon^{2-2(m+1) \kappa} \exp \left(2 c\left(1+\ln \left(\varepsilon^{-\frac{1}{\zeta}}\right)\right) T_{0}\right) \\
& \leq C e^{2 c T_{0}} \varepsilon^{2-2(m+1) \kappa-2 \tilde{\kappa}}
\end{aligned}
$$

where $\tilde{\kappa}=\frac{c T_{0}}{\zeta}$. If we choose $\tilde{\kappa} \leq \kappa$ for sufficiently large $\zeta$, then $|Q(t)|<1$ for $\kappa<\frac{1}{m+2}$ and small $\varepsilon$. Taking supremum on $\left[0, \tau^{*} \wedge T_{1}\right]$

$$
\sup _{t \in\left[0, \tau^{*} \wedge T_{1}\right]}|Q(t)| \leq C \varepsilon^{1-(m+2) \kappa} \text { on } \stackrel{* *}{\Omega} .
$$

Hence,

$$
\begin{aligned}
\sup _{\left[0, \tau^{*} \wedge T_{1}\right]}|a-b| & =\sup _{\left[0, \tau^{*} \wedge T_{1}\right]}\left|Q-R^{(2)}\right| \leq \sup _{\left[0, \tau^{*} \wedge T_{1}\right]}|Q|+\sup _{\left[0, \tau^{*} \wedge T_{1}\right]}\left|R^{(2)}\right| \\
& \leq C \varepsilon^{1-(m+2) \kappa} \text { on } \stackrel{*}{\Omega} .
\end{aligned}
$$


Now we can use the results obtained previously to prove the main result of Theorem 20 and Corollary 21] for the SPDE (11).

Proof of Theorem 20. Similar steps than the proof of Theorem 17.

Proof of Corollary 21, Similar steps than the proof of Corollary 18.

\subsection{Application of Approximation Theorem II}

In this subsection we apply our main Theorem 20 to the nonlinear heat equation (83) and a cubic auto-catalytic reaction (85) with $\sigma_{\varepsilon}=1$ and non-zero $\alpha_{k, 0}$.

\subsubsection{Physical Application (Nonlinear Heat Eq.)}

Our main Theorem 20] in this case states that the solution of (83) takes the form

$$
u(t)=b(t)+\mathcal{O}\left(\varepsilon^{1-}\right),
$$

where $b$ is the solution of stochastic ordinary differential equation

$$
d b=\left[b-b^{3}\right] d t+d B,
$$

and $B$ is a $\mathbb{R}$-valued standard Brownian motion given by

$$
B(t)=\alpha_{1,0} \beta_{1,0}(t)+\alpha_{2,0} \beta_{2,0}(t)+\alpha_{3,0} \beta_{3,0}(t)+\alpha_{4,0} \beta_{4,0}(t) .
$$

To check the bound on $b$ consider $\exp \left\{\delta|b|^{2}\right\}$. We note that

$$
d \exp \left\{\delta|b|^{2}\right\}=\delta \exp \left\{\delta|b|^{2}\right\} d|b|^{2}+\delta^{2} \exp \left\{\delta|b|^{2}\right\}\left(d|b|^{2}\right)^{2},
$$

and

$$
d|b|^{2}=2 b \cdot d b+d b \cdot d b .
$$

From (94) we obtain for some constant $c>0$

$$
\begin{aligned}
d|b|^{2} & =2|b|^{2} d t-2|b|^{4} d t+2 b \cdot d B+d B \cdot d B \\
& =\left(C+2|b|^{2}-2|b|^{4}\right) d t+2 b \cdot d B .
\end{aligned}
$$

Substituting this into (95), yields

$$
\begin{aligned}
d \exp \left\{\delta|b|^{2}\right\} & =\delta\left(C+(2+4 \delta)|b|^{2}-2|b|^{4}\right) \exp \left\{\delta|b|^{2}\right\} d t+2 \delta \exp \left\{\delta|b|^{2}\right\} b \cdot d B \\
& \leq c_{\delta} \exp \left\{\delta|b|^{2}\right\} d t+2 \delta \exp \left\{\delta|b|^{2}\right\} b \cdot d B .
\end{aligned}
$$

Integrating from 0 to $t$ and taking expectation, yields

$$
\mathbb{E} \exp \left\{\delta|b(t)|^{2}\right\} \leq \mathbb{E} \exp \left\{\delta|b(0)|^{2}\right\}+c_{\delta} \int_{0}^{t} \mathbb{E} \exp \left\{\delta|b|^{2}\right\} d t
$$

As $\mathbb{E} \exp \left\{3 \delta|b(0)|^{2}\right\} \leq C$ and applying Gronwall's lemma, yields for $t \leq T_{1}$

$$
\sup _{\left[0, T_{1}\right]} \mathbb{E} \exp \left\{\delta|b|^{2}\right\} \leq C \text {. }
$$

With $3 \delta$ instead of $\delta$, we have

$$
\sup _{\left[0, T_{1}\right]} \mathbb{E} \exp \left\{3 \delta|b|^{2}\right\} \leq C .
$$


Taking expectation after supremum on both sides of (96) to obtain

$$
\begin{aligned}
& \left.\mathbb{E} \sup _{t \in\left[0, T_{1}\right]} \exp \left\{\delta|b(t)|^{2}\right\}\right) \\
& \leq \mathbb{E} \exp \left\{\delta|b(0)|^{2}\right\}+c_{\delta} \mathbb{E} \sup _{t \in\left[0, T_{1}\right]} \int_{0}^{t} \exp \left\{\delta|b(s)|^{2}\right\} d s \\
& \quad+2 \delta \mathbb{E} \sup _{t \in\left[0, T_{1}\right]} \int_{0}^{t} b(s) \exp \left\{\delta|b(s)|^{2}\right\} d B(s) \\
& \leq \quad C+c_{\delta} \mathbb{E} \int_{0}^{T_{1}} \exp \left\{\delta|b(s)|^{2}\right\} d s+2 \delta \mathbb{E}\left(\int_{0}^{T_{1}} b(s)^{2} \exp \left\{2 \delta|b(s)|^{2}\right\} d s\right)^{1 / 2} .
\end{aligned}
$$

Using (98)) together with $x e^{2 \delta x} \leq C e^{3 \delta x}$ for all $x>0$, yields

$$
\mathbb{E} \sup _{t \in\left[0, T_{1}\right]} \exp \left\{\delta|b(t)|^{2}\right\} \leq C .
$$

Now, using Chebychev inequality

$$
\mathbb{P}\left(\sup _{\left[0, T_{1}\right]}|b(t)|^{2}>\ln \left(\varepsilon^{-\kappa}\right)\right) \leq \frac{\mathbb{E} \sup _{t \in\left[0, T_{1}\right]} \exp \left(\delta|b(t)|^{2}\right)}{\exp \left(\delta \ln \left(\varepsilon^{-\kappa}\right)\right)} \leq C \varepsilon^{\delta \kappa} .
$$

\subsubsection{Chemical Application}

Our main theorem states that the solution of (85) takes the form

$$
\begin{gathered}
u(t)=b(t)+\mathcal{O}\left(\varepsilon^{1-}\right), \\
\text { with } u=\left(\begin{array}{l}
u_{1} \\
u_{2}
\end{array}\right) \text { and } b=\left(\begin{array}{l}
b_{1} \\
b_{2}
\end{array}\right) .
\end{gathered}
$$

In this case $b_{1}$ and $b_{2}$ are the solutions of

$$
d b_{1}=-\rho b_{1} b_{2}^{2} d t+d B_{1}(t) \quad \& \quad d b_{2}=\rho b_{1} b_{2}^{2} d t+d B_{2}(t),
$$

where

$$
B_{i}(t)=\alpha_{i_{1}, 0} \beta_{i_{1}, 0}+\alpha_{i_{2}, 0} \beta_{i_{2}, 0}(t)+\alpha_{i_{3}, 0} \beta_{i_{3}, 0}(t)+\alpha_{i_{4}, 0} \beta_{i_{4}, 0}(t) \text { for } i=1,2 .
$$

To verify the bound on $b$ define first the stopping $T_{1}$ as

$$
T_{1}=T_{0} \wedge \inf \left\{t>0: \exists i \in\{1,2\}: b_{i}(t)<0\right\} .
$$

This means that our approximation result is only true as long as the concentrations $b_{i}$ are non-negative.

Now, we note that

$$
\sum_{i=1}^{2} d b_{i}=\sum_{i=1}^{2} d B_{i}
$$

Integrating from 0 to $t$, yields

$$
\sum_{i=1}^{2} b_{i}(t)=\sum_{i=1}^{2} b_{i}(0)+\sum_{i=1}^{2} B_{i}(t)
$$


Hence, up to $T_{1}$ we obtain

$$
|b(t)| \leq \sum_{i=1}^{2} b_{i}(t)=\sum_{i=1}^{2} b_{i}(0)+\sum_{i=1}^{2} B_{i}(t) \leq \sqrt{2}|B(t)|+\sqrt{2}|b(0)|,
$$

where we used $\left(x^{2}+y^{2}\right)^{1 / 2} \leq|x|+|y| \leq \sqrt{2}\left(x^{2}+y^{2}\right)^{1 / 2}$. Moreover,

$$
|b(t)|^{2} \leq 4|B(t)|^{2}+4|b(0)|^{2} .
$$

Thus

$$
\mathbb{E} \sup _{\left[0, T_{1}\right]} \exp \left\{\delta|b|^{2}\right\} \leq \mathbb{E} \sup _{\left[0, T_{1}\right]} \exp \left\{4 \delta|B|^{2}\right\} \cdot \exp \left\{4 \delta|b(0)|^{2}\right\} \leq C,
$$

but only for sufficiently small $\delta$. Using Chebychev inequality

$$
\mathbb{P}\left(\sup _{\left[0, T_{1}\right]}|b(t)|^{2}>\ln \left(\varepsilon^{-\kappa}\right)\right) \leq \frac{\mathbb{E} \sup _{t \in\left[0, T_{1}\right]}\left(\exp \left(\delta|b(t)|^{2}\right)\right)}{\exp \left(\delta \ln \left(\varepsilon^{-\kappa}\right)\right)} \leq C \varepsilon^{\delta \kappa} .
$$

So the probability is close, but not very close to 1 , as $\delta$ cannot be arbitrarily large.

\section{Acknowledgements}

This work was supported by the Deutsche Forschungsgemeinschaft (DFG) 'Multiscale Analysis of SPDEs' (DFG BL535/9-2).

\section{References}

[1] E. Alós and S. Bonaccorsi. Stochastic partial differential equations with Dirichlet white-noise boundary conditions Annales de l'institut Henri Poincaré (B) Probabilits et Statistiques, 38(2):125-154, (2002)

[2] D. Blömker and J. Duan. Predictability of the Burgers dynamics under model uncertainty, Stochastic Differential Equations: Theory and Applications, P. Baxendale and S. Lototsky (Eds.), p.71-90, World Scientific, New Jersey, 2007.

[3] Z. Brzezniak, B. Goldys, S. Peszat and F. Russo. Second Order PDEs with Dirichlet White Noise Boundary Condition. J. Evol. Equ. (to appear).

[4] S. Cerrai and M. Freidlin. Fast transport asymptotics for stochastic RDE's with boundary noise, Ann. Probab. 39:369-405, (2011).

[5] R. Courant and D. Hilbert. Methoden der mathematischen Physik. (Methods of mathematical physics). 4. Aufl. (German) Springer, (1993).

[6] D. Grieser. Uniform bounds for eigenfunctions of the Laplacian on manifolds with boundary, Commun. Partial Diff. Eq., 1283-1299, (2002).

[7] G. Da Prato and J. Zabczyk. Stochastic equations in infinite dimensions. Vol. 44 of Encyclopedia of Mathematics and its Applications. Cambridge University Press, Cambridge, (1992). 
[8] G. Da Prato and J. Zabczyk. Evolution equations with white-noise boundary conditions. Stochastics Stochastics Rep. 42:167-182, (1993).

[9] G. Da Prato and J. Zabczyk. Ergodicity for infinite-dimensional systems, volume 229 of London Mathematical Society Lecture Note Series. Cambridge University Press, Cambridge, (1996).

[10] D. Henry. Geometric theory of semilinear parabolic equations, Lecture Notes in Mathematics 840, Springer-Verlag, Berlin, (1981).

[11] R. E. Langer. A problem in diffusion or in the flow of heat for a solid in contact with a fluid. Tohoku Math. J. 35:260-275, (1932).

[12] L. Lapidus and N. Amundson. Chemical reactor theory, Prentice-Hall, (1977).

[13] B. Maslowski, Stability of semilinear equations with boundary and pointwise noise, Ann. Scuola Norm. Sup. Pisa Cl. Sci., 22(4):55-93, (1995).

[14] T. Runst and W. Sickel. Sobolev spaces of fractional order, Nemytskij operators, and nonlinear partial differential equations. Walter de Gruyter. Berlin. New York, (1996).

[15] R.B. Sowers, Multidimensional reaction-diffusion equations with white noise boundary perturbations, Ann. Probab. 22:2071-2121, (1994)

[16] A. Pazy. Semigroups of linear operators and applications to partial differential equations. Applied Mathematical Sciences, 44. New York etc.: Springer-Verlag (1983).

[17] J. P. Peixoto and A. H. Oort. Physics of climate. Springer, New York, (1992).

[18] R. Schnaubelt, M. Veraar, Stochastic Equations with Boundary Noise. Parabolic Problems. Progress in Nonlinear Differential Equations and Their Applications, 80:609-629, (2011).

[19] R. Vold and M. Vold. Colloid and interface chemistry, Addison-Wesley, (1983). 